Aus dem Anatomischen Institut zu Greifswald.

\title{
Die Bedeutung von sauren Kolloiden und Lipoiden für die vitale Farbstoffbindung in den Zellen.
}

\author{
Von \\ Wilhelm von Moellendorff. \\ (Abgeschlossen Oktober 1916.)
}

Inhalt:

1. Einleitung . . . . . . . . . . . . . . . . . . . 503

2. Über die Granulafärbung entscheidet die Fällungskraft nicht allein . 505

a) Der Einfluss der Farbstoffkonstitution auf die Granulafärbung 505

b) Direkte Messung der Fällungskraft basischer gegenüber sauren Farbstoffen . . . . . . . . . . . . . . . 508

3. Die Diffusfärbung der Grundmasse des Protoplasmas in supravitalen

Versuchen . . . . . . . . . . . . . . . . . . . 510

a) Die Tatsachen . . . . . . . . . . . . . . . . . . . . . . 510

b) Der Grad der Diffusfärbung ist von der Dispersität der Farbstoffe nicht abhängig; das Problem der Zellpermeabilität . . 515

c) Die Diffusfärbung ist von den Zellipoiden abbängig . . . . . 521

4. Die Lipoidlöslichkeit verschlechtert die Elektivität der Granulafärbung durch basische Farbstoffe . . . . . . . . . . . . . . . . 533

5. Zusammenfassung und Zusätze . . . . . . . . . . . . . . . 539

6. Literaturverzeichnis . . . . . . . . . . . . . . . . . . 541

\section{Einleitung.}

In der vorangehenden Arbeit in dieser Zeitschrift habe ich zu begründen versucht, dass die vitale und supravitale Färbung mit basischen Farbstoffen als Reaktion des basischen Farbstoffes mit sauren, granulär in den Zellen abgelagerten Substanzen aufzufassen sei. Die Gründe, die ich in der erwähnten Mitteilung anführte, waren morphologischer und experimenteller Art. Morphologisch verläuft der Vorgang der Granulafärbung unter Fällungserscheinungen, die besonders gut an den grossen Granulis embryonaler Amphibienzellen beobachtet werden können, aber auch $\mathrm{z}$. B. an den Granulis von Zellen der Mäuseniere deutlich werden. Es 
konnte ferner gezeigt werden, dass die Anfärbung saurer Farbstoffgranula unter den gleichen Erscheinungen abläuft, wie die der zelleigenen Granula, dass somit die sauren Farbstoffgranula geradezu als analoge Bildungen mit den basisch färbbaren Zellgranulis aufzufassen sind. Es wurde daraus geschlossen, dass zum Zustandekommen der basischen Granulafärbung besondere vitale Eigenschaften der Granula nicht erforderlich sind, dass vielmehr nur das Vorhandensein eines sauren reaktionsfähigen Körpers in den Granulis Voraussetzung für die Vereinigung mit dem basischen Farbstoff ist. In einem Falle ist dieser Körper (als saurer experimentell eingelagerter Farbstoff) bekannt, seine Beziehungen zu dem zur Färbung gewăhlten basischen Farbstoff also in vitro erforschbar; die Eigenschaften der zelleigenen Granula dagegen lassen sich aus der gleichartigen Reaktionsweise auf denselben basischen Farbstoff bis zu einem gewissen Grade erschliessen. Die vitale Ablagerung saurer Farbstoffe in den Zellen ist daher geeignet, ein sehr brauchbares Mittel zur Erforschung der 7ellgranula zu werden.

Zweifellos ist die Parallele zwischen der Färbbarkeit der Zellgranula und der künstlich in den Zellen abgelagerten sauren Farbstoffe überraschend vollkommen; das lehrt die in der erwähnten Mitteilung auf seite 489 abgedruckte Tabelle. Es war damit die Möglichkeit gegeben, die Art der Reaktionsweise der basischen Farbstoffe mit den Zellgranulis näher zu erforschen.

Eine kurze Überlegung und die Betrachtung der bereits bekannten Tatsachen über die Wirkungsweise der Farbstoffe zeigt, dass abgesehen von den Eigenschaften der beiden miteinander reagierenden Substanzen (basischer Farbstoff und saurer Farbstoff, resp. saure Granulasubstanz) noch mindestens e in besonderer Faktor zum Zustandekommen der vitalen Granulafärbung notwendig ist. Denn eine der eigenartigsten Seiten dieses Vorganges ist es ja, dass unter einer grossen Zahl bisher versuchter Farbstoffe sich nur relativ wenige als geeignet herausgestellt haben. Ehe also nicht gefunden ist, wovon die Reaktionsweise der basischen Farbstoffe mit den Zellgranulis in so vielen Fällen ungünstig beeinflusst wird, kann die bisher hauptsächlich morphologisch erschlossene Ansicht von der Bedeutung kolloidchemischer Reaktionen bei dem Zustandekommen der vitalen Granulafärbung nicht als bewiesen gelten. 
Es ist also die schon vielfach aufgeworfene, bislang aber nicht befriedigend gelöste Frage, welchen Eigenschaften unsere guten vitalen Granulafarbstoffe diese ihre Eignung verdanken, die ich durch meine Versuche einer Lösung näher zu bringen hoffe.

\section{II. Über die Granulafärbung entscheidet die Fällungs- kraft nicht allein.}

a) Der Einfluss der Farbstoffkonstitution auf die Granulafärbung.

Nachstehend sind die von mir verwandten basischen Farbstoffe mit den Molekulargewichten aufgeführt, die nach den Angaben in den Farbstofftabellen von Schulz und Julius berechnet wurden.

\section{Triphenylmethanfarbstoffe}

Diamantfuchsin kl. Krist. (Bayer) . . . . . . . . . . . ca. 409

Rosanilin Base (Kalle \& Co.) . . . . . . . . . . . . . . ca. 344

Auramin konz. (Höchster Farbw.) . . . . . . . . . . . . 303

Methylviolett 5 B (Bayer) . . . . . . . . . . . . . . . 392

Kristallviolett 6B Pulver (Akt.-Ges. f. Anil.-Fabr.) . . . . 407

Malachitgrün Krist. chem. rein (Höchst. Farbw.) . . . . . 363

\section{Xanthonfarbstoffe}

Irisamin G extra (Bad., Anil.-Fabr.) . . . . . . . . . . 541

Acridinrot 3 B (Leonhardt) . . . . . . . . . . 274

Rhodamin S extra (Bad. Anil.-Fabr.) . . . . . . . . . . 374

$\quad G$ extra (Kahlbaum) . . . . . . . . . . 407

, B ( , . . . . . . . . 478

,$\quad$ B extra (Bad. Anil.-Fabr.) . . . . . . . . . 478

$\Rightarrow 3 \mathrm{~B}$ extra ( $\pi) . .506$

Azine

Indazin M (Cassella \& Co.) . . . . . . . . . . . . . . . 493

Neutralrot (Gruebler) . . . . . . . . . . . . . 287

Safranin G extra (Bad. Anil.-Fabr.) . . . . . . . . ca. 3557

Basler Blau R (Durand \& Huguenin) . . . . . . . . . . . 432

Basler Blau BB (Durand \& Huguenin) . . . . . . . . . . . . 404

Azofarbstoffe

Bismarckbraun (Gruebler) . . . . . . . . . . 334

Vesuvin 4BG (Höchster Farbw.) . . . . . . . . . . . 227

Chrysoidin R (Durand \& Huguenin) . . . . . . . . . . . 248

Oxazine

Nilblausulfat (Gruebler) . . . . . . . . . . . . . . . . 349

Nilblauchlorhydrat (Gruebler) . . . . . . . . . . . . . . . . 437

Capriblau GON (Bayer) . . . . . . . . . . . . 317 
Thiazine

Toluidinblau (Gruebler). . . . . . . . . . . . 306

Methylengrün extra gelbl. 0 (Höchster Farbw.) . . . . . . . 409

Methylenblau BB (Bayer). . . . . . . . . . . . . 393

Methylenblan rectif. (Gruebler) . . . . . . . . . . 393

Methylenblau BX (Gruebler) . . . . . . . . . . . . . 393

Diphenylnaphthylmethanfarbstoffe

Viktoriablau B (Bad. Anil. Fabr.) . . . . . . . . . . 505

\begin{tabular}{|c|c|}
\hline $\begin{array}{l}R \\
4 R S\end{array}$ & $n$ \\
\hline
\end{tabular}

Den hier genannten Firmen bin ich für die freundliche Überlassung von Proben dieser sowie vieler anderer Farbstotfe den grössten Dank schuldig.

Die Untersuchung der Konstitution der genannten Farbstoffe fübrte $z u$ keinem befriedigenden Ergebnis; ich konnte im wesentlichen die Angaben von Fischel bestätigen. Besonders ergibt sich die Verstärkung der granulafürbenden Eigenschaften durch Substitution des Wasserstoffrestes in der Ammoniakgruppe durch Alkoholradikale; unter den Triphenylmethanfarbstoffen liegen die Verhältnisse folgendermassen:

\begin{tabular}{|c|c|c|}
\hline Farbstoff & anxochrome Gruppen & Art der Färbung \\
\hline $\begin{array}{l}\text { Diamantfuchsin } \\
\text { Rosanilin Base } \\
\text { Auramin konz. } \\
\text { Methylviolett } \\
\text { Kristallviolett } \\
\text { Malachitgrün }\end{array}$ & $\begin{array}{l}3 \mathrm{NH}_{2} \text {-Gruppen } \\
1 \mathrm{HN}_{2}, 2 \mathrm{~N}\left(\mathrm{CH}_{3}\right)_{2} \\
1 \mathrm{NH}\left(\mathrm{CH}_{3}\right), 2 \mathrm{~N}\left(\mathrm{CH}_{3}\right)_{2} \\
3 \mathrm{~N}\left(\mathrm{CH}_{3}\right)_{2} \\
2 \mathrm{~N}\left(\mathrm{CH}_{3}\right)_{2}\end{array}$ & $\begin{array}{l}\text { Keine bis scblechte } \\
\text { Granulafärbung } \\
\text { mässiger Granulafärber } \\
\text { gute Granulafärber }\end{array}$ \\
\hline
\end{tabular}

Ebenso ist wohl in der Reihe der Thiazine die Verbesserung der Granulafärbung mit zunehmender Substitution aufzufassen. Thionin (mit $2 \mathrm{NH}_{2}$ ) farbt schlecht, Toluidinblau $\left(\mathrm{NHCH}_{3}, \mathrm{~N}_{\left.\left(\mathrm{CH}_{3}\right)_{2}\right)}\right.$ und Methylenblau, Metbylengrün $\left(2 \mathrm{~N}\left(\mathrm{CH}_{3}\right)_{2}\right.$ sind gute Granulafärber.

Die Wirkung der auxochromen Gruppen darf aber nicht verallgemeinert werden. Zahlreiche Farbstoffe, die eine gute Substitution ihrer Ammoniakgruppen zeigen, sind trotzdem nur schlechte Granulafärber. Hierher gehören z. B. sämtliche Rhodamine. Die Feststellung von $\mathrm{F}$ is $\mathrm{chel}$, dass die Substitution eines $H$ durch einen Anilinrest das Färbevermögen aufhebe, könnte vielleicht für Basler Blau $\mathrm{R}$ und $\mathrm{BB}$ zur Erklärung ihrer schlechten 
Fårbeergebnisse herangezogen werden; doch lässt vor allem die schlechte Wasserlöslichkeit die Färbung dieser Stoffe schwer beurteilen. Andererseits ist Indazin M ein Granulafärber, trotzdem es ein Gemenge aus

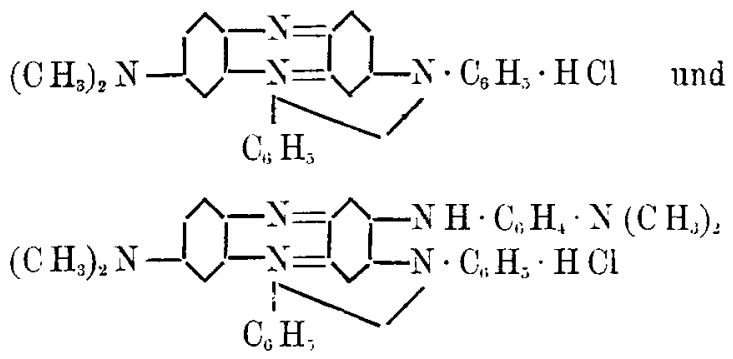

darstellt, ebenso wissen wir ja aus den Untersuchungen von M ic ha elis, Laguesse u. a., dass Janusgrün trotz der safraninartigen Konstitution als Granulafürber zu betrachten ist. Endlich sei darauf hingewiesen, dass Viktoriablau, wie schon $\mathrm{H}$ o eber zeigte, Granula $\mathrm{zu}$ farben imstande ist. Viktoriablau B besitzt ebenfalls, wie auch $\mathrm{F}$ isch el bervorhebt, ein durch den Anilinrest substitutiertes Ammoniakradikal. Damit muss man wohl die von $\mathrm{F}$ is chel an seinem IIaterial erhobene Feststellung, dass die Substitution durch den Anlinrest das Färbevermögen verhindert, fallen lassen.

Weiter ist helvolzuheben, dass auch Farbstofie, die nur einfache $\mathrm{NH}_{2}$-Gruppen entbalten, als Granulafarbstoffe zu bezeichnen sind; so besonders die Azofarbstoffe Chrysoidin $\mathrm{R}$ mit 2, Vesuvin $4 \mathrm{BG}$ mit 3 und Bismarckbraun mit $4 \mathrm{NH}_{2}$-Gruppen.

Aus diesen Betrachtungen geht nur soviel hervor, dass bei gewissen Farbstoffgruppen, die in ihrem Molekül den gleichen Grundstock besitzen, das Hinzutreten bestimmter auxochromer Gruppen, so besonders der wachsenden Substitution der Ammoniakreste durch Alkoholradikale, die Tendenz zur Granulafärbung in bestimmter Weise beeinflusst. Darüber hinaus spielen aber andere, noch nicht $\mathrm{zu}$ übersehende Einflüsse der Konstitution des Farbstoffmoleküls eine Rolle. Sie zu übersehen, muss immer noch künftiger Forschung vorbehalten bleiben. Vor allem erscheint es notwendig, Versuchsreihen, wie die hier mitgeteilten, mit reinen Farbstoffen anzustellen, da ja sicherlich die vielfachen Zusätze and Verunreinigungen der hier ausschliesslich verwandten technischen Farbstoffe für die Beurteilung sehr störend sein müssen. 


\section{b) Direkte Messung der Füllungskraft basischer gegenüber sauren Farbstoffen.}

Da also durch die Betrachtung der Strukturformeln ein $\mathrm{Zu}$ sammenhang zwischen einer Struktureigenart und dem Vermögen der Farbstoffe, mit den Granulis zu reagieren, nicht erkannt werden konnte, wählte ich den direkteren Weg, indem ich die Reagierfähigkeit der basischen Farbstoffe mit den verschiedenen zu den Versuchen verwandten sauren Farbstoffen im Reagenzglas prüfte.

Alle sauren Farbstoffe lassen sich in vitro durch basische Farbstoffe făllen; bei der Bildung der Neutralfarbe tritt eine messbare Wärmeentwicklung auf (Seyewetz). Im allgemeinen reagieren die basischen Farbstoffe mit sauren im Mengenverhältnis 3:1,2: 1oder $1: 1$ (Vaubel und $\mathrm{Bartlet}$ ). Ich habe allerdings bei meinen zahlreichen Versuchen auch ganz andere Mengenverhaltnisse angetroffen.

So reagiert Trypanbla u mit 9 Molekülen $d k r i d i n r o t$ $3 \mathrm{~B}$, mit 15 Mol. Rhodamin 0 .

Vaubel und Bartlet fanden, dass die Zabl der Sulfosäuregruppen in sauren Farbstoffen eine gewisse Bedeutung für die Reaktion mit basischen Farbstoffen besitzt. Ist nur eine Sulfogruppe vorhanden, so fixiert sich nur ein Methylenblaurnolekül, bei Anwesenbeit mebrerer Sulfogruppen zwei.

"Aus der Gesamtheit dieser Tatsachen ist zu scbliessen. dass die sauren Farbstoffe sich mit den basischen verbinden. indem sie Salze bilden, die übrigens wenig beständig und leicht spaltbar sind." (Pelet-Jolivet, Theorje des Färbeprozesses Seite 43 , dessen Darstellung ich auch sonst hier folge.)

Auf die Ausfällung des Reaktionsproduktes ist der Kolloidzustand der miteinander reagierenden Farbstoffe von bedeutendem Einflusse (Teague und Buxton, 1907).

Ich habe nach der Methodik von Pelet-Jolivet eine grosse Anzahl von Farbstoffpaaren untersucht.

$\mathrm{Zu}$ den Versuchen wurden $1 / 200$ Normallösungen der Farbstoffe verwandt; von der Lösung des basischen Farbstoffes wurde zu der des sauren vorsichtig so lange hinzugemischt, bis eine möglichst vollständige Ausfällung des Neutralproduktes erreicht war. Bei manchen Farbstoffkombinationen wurde ein scharfer Nentralpunkt nicht erreicht, besonders, wenn der basische Farbstoff sehr diffusibel war. Zur Kontrolle wurde jeweils ein Tropfen der Mischung auf Fliesspapier aufgetropft; der Überschuss eines der beiden Farb- 
stoffe kommt dann durch Ringbildung der betreffenden Farbe um den durch das Fällungsprodukt gebildeten Fleck herum zum Ausdruck. Hält, wie es z. B. beim Überschuss eines hochkolloidalen Farbstoffes die Regel ist, der Überschuss dieses Farbstoftes einen Teil des Fällungsprodultes in Lösung, so entstehen hỉnfig drei Zonen: in der Mitte das ausgefallene Produkt, das gelöste Fällungsprodukt als Ring in der Mischfarbe und aussen die reine Farbe des im Überschuss befindlichen Farbstoffes.

Für Trypanblau erhielt ich anf diese Weise folgende Ergebnisse :

1 Mol Trypanblau wird gefüllt durch

1 Hol von Neutralrot, Diamantfuchsin, Chrysoidin, Malachitgrun, Toluidinblau,

2 Mol von Auramin, Rhodamin S extra, Safranin B extra, Methylviolett.

3 Mol von Bismarckbraun, Kristallviolest, Methylengrün, Rhodamin B extra.

Schon dieses eine Beispiel zeigt, dass die Erwartung, dass etwa die Fillungskraft der basischen Farbstoffe die Erklärung für ihre verschiedenartige Eignung $7 u$ einer Anfärbung saurer Substanzen im biologischen Versuche abgeben könnte, nicht zutrifftt. Man hätte ja denken können, dass Farbstoffe, bei denen schon 1 Mol genügt, um eine entsprechende Menge sauren Farbstoffes auszufallen, bessere Granulafärber sein müssten, als solche basische Farbstoffe, von denen hierzu 2 oder $3 \mathrm{Mol}$ notwendig sind. Dagegen lehrt der Vergleich der obigen Zahlen mit dem Ergebnis der supravitalen Färbungsversuche, dass in allen drei Gruppen sowohl gute als auffallend schlechte Granulafärber enthalten sind.

Gute Granulafärber sind: Neutralrot, Malachitgrün, Toluidinblau, Auramin, Methylviolett, Bismarckbraun, Kristallviolett, Methylengrün, ganz schlecht färben dagegen Granula: Diamantfuchsin. Rhodamin S extra. Safranin B extra, Rhodamin B extra

Es war daher von vornherein kiar, dass zur Erklärung der verschiedenartigen Ergebnisse der biologischen Versuche bei den einzelnen basischen Farbstoffen noch andere Gesichtspunkte angewandt werden mussten. Ich will bier nur darauf hinweisen, dass auch für andere saure Farbstoffe ihre Fällbarkeit durch basische Farbstoffe untersucht wurde, mit dem gleichen Ergebnis, dass Fallbarkeit im Reagenzglas und im biologischen Versuche nicht miteinander übereinstimmten. 


\section{Die Diffusfärbung der Grundmasse des Proto- plasmas in supravitalen Versuchen.}

\section{a) Die Tatsachen.}

Trotzdem also die morphologische Betrachtung der granulären Färbung in ibrem Zustandekommen und die Möglicbkeit, dieselben Färbungseffekte mit basischen Farbstoffen auch dann zu erzielen, wenn saure Farbstoffe den wesentlichen Bestandteil des Granulums bilden, trotzdem also diese Tatsachen durchaus für das reaktive Zustandekommen der basischen Granulafärbung sprachen, erweist sich die rein chemische Beurteilung der Frage nach der Elektivität gewisser Farbstoffe, nach deren Ursachen als völlig unzureichend.

Sollte trotzdem an der Deutung der Granulafärbung als eines Reaktionsvorganges festgehalten werden, so musste nach den Gründen geforscht werden, die für die Behinderung eines an sich gut reaktionstähigen basischen Farbstoffes, die Granula zu fárben, verantwortlich sein können.

Wo diese Gründe zu suchen seien, war nur auf dem Wege des Experiments zu ergründen. Es war naheliegend anzunehmen, dass vor allem andere Teile der Zelle einen solchen hindernden Einfluss auf bestimmte F'arbstoffe auszuüben imstande sind. Diese Annahme war um so naheliegender, als ein Einfluss und eine rege Anteilnahme des lebenden und überlebenden Protoplasmas an dem Vorgang der vitalen Granulafärbung für bestimmte Falle sogar schon einigermassen analysiert ist. Ich erinnere hier an die schon lange zurückliegenden Beobachtungen über die Färbbarkeit (phagozytierter Massen) Br. H of er 1891, A. P l a to 1900); hier ergab sich, dass Infusorien, Bakterien und andere phagozytable Vassen, die im freien Zustande nur schlecht oder garnicht farbbar waren, nach der Aufnahme in den Protoplasmaleib von Protozoen oder Leukozyten intensiv durch basische Farbstoffe gefärbt werden konnten. Plato konnte sogar zeigen, dass eine solche Färbung nur im Endoplasma eintritt, während Gonokokken im Exoplasma die Farbe wieder abgaben. E. Nirenstein konnte nachweisen, dass die Fürbung der Nahrungsmassen von der Produktion von Săure seitens des Protoplasmas abhängig ist.

Wie sich in diesen Fällen ein Einfluss der Protoplasmatätigkeit auf die vitale Färbbarkeit beobachten lässt, so sind die FinHüsse des lebenden Protoplasmas zum Teil sicher in ihrer Trag- 
weite noch gar nicht $\mathrm{zu}$ übersehen. Ich will hier nur an die Möglichkeit erinnern, dass das Protoplasma eine reduzierende oder oxydierende Wirkung aut Farbstoffe ausüben kann.

Wenig beachtet ist dagegen bisher die Tatsache, dass eine ganze Reihe von Farbstoffen, denen offenbar die Fähigkeit, Granula zu färben, abgeht, trotzdem eine Färbung in den Zellen hervorbringen, die anscheinend diffus den ganzen Zelleib durchtränkt, ohne dass einzelne Gebilde stärker gefarbt würden.

Dass wir es hier mit einer für die Farbstoffwirkung charakteristischen Tatsache zu tun haben, lehrt ein kurzer Überblick über meine Ergebnisse an supravitalen Versuchen.

Über die Technik diesel Versuche siehe die vorhergehende Arbeit Seite 486.

Die folgende Tabelle führt die untersuchten Farbstoffe auf; sie sind geordnet nach ihrer Fahigkeit, das Zellenprotoplasma diffus $z u$ färben; gleichzeitig sind in einer dritten Kolumne die Angaben aus meiner eingangs erwahnten Arbeit über die Güte der Granulafürbung wiedergegeben. Die Tabelle ist das Ergebnis zahlreicher Versuche; die Diffusfärbung bildet sich bei jedem basischen Farbstoff in allen Versuchen in der gleichen, angegebenen charakteristischen Weise aus, ganz gleich, ob das Versuchstier vor dem Versuch unbehandelt war, oder ob vorher durch saure Farbstoffe eine Granulabildung in den beobachteten Zellen hervorgerufen worden war.

Das Ergebnis der Tabelle stimmt mit den allgemeinen Erfahrungen gut überein; die Tendenz zur Diffusfärbung ist im allgemeinen um so stärker, je weniger gut eine Granulafärbung $z u$ erzielen ist. Der Begriff "Diffusfürbung" ist morphologisch schwer exakt zu definieren. Er kann nur ausdrücken, dass im Gegensatz zu anderen Färbungen, bei denen wohl charakterisierte Zellelemente gefärbt werden, hier eine mehr allgemeine Färbung des Zellprotoplasmas gefunden wird. Es ist dabei möglich, dass entweder eine Lösung des Farbstoffes in der Grundsubstanz des Protoplasmas (dem Dispersionsmittel, wenn man das Protoplasma im Sinne Lepeschkins 1913 als eine emulsionskolloide Lösung betrachtet) stattfindet, oder dass der Farbstoff sich durch Adsorption oder chemische Affinität an kleine Körperchen von ultramikroskopischen Dimensionen anlagert. Über die Art der Farbstoffbindung an das Protoplasma gibt eben die Betrachtung 


\begin{tabular}{|c|c|c|c|c|c|}
\hline Farbstoff & $\begin{array}{l}\text { Grad der } \\
\text { Dlffus- } \\
\text { farbung }\end{array}$ & $\left|\begin{array}{c}\text { fărot Granula } \\
\text { beim } \\
\text { normalen } \\
\text { Tier }\end{array}\right|$ & $\begin{array}{l}\text { Konzen- } \\
\text { tration }\end{array}$ & $\begin{array}{l}\text { Wasser- } \\
\text { löslichkeit }\end{array}$ & $\begin{array}{c}\text { I ccm } 2 \% \text { Lezi- } \\
\text { thinxylol absorbiert } \\
\text { aus io ccm } \\
\text { der } n / 10000 \text { Lsg. }\end{array}$ \\
\hline $\begin{array}{l}\text { Safrauin G extra } \\
\text { Rhodamin G }\end{array}$ & stark & nicht & n. 10000 & gut & n. 4000 \\
\hline extra & $\Rightarrow$ & fraglich & $\mathrm{n} / 10000$ & $\because$ & $\mathbf{n} / \mathbf{5 0 0 0}$ \\
\hline Rosanilin Base & $"$ & $\begin{array}{l}\text { schlecht } \\
\text { nicht }\end{array}$ & $\begin{array}{c}n / 100010 \\
n, 1000\end{array}$ & schlecht & $\begin{array}{l}\text { ni } 10000 \\
\text { n } 10000\end{array}$ \\
\hline Kristallviolett & , & mittel & $\mathrm{n} / 10000$ & gut & $\mathbf{n}_{i} 10000$ \\
\hline Methylviolett & " & mittel & $\mathrm{n}_{i} 10000$ & $\Rightarrow$ & $\mathrm{n}_{1} 10000$ \\
\hline Irisamin G extra & $"$ & fraglich & n 10000 & 》 & n $1 \overline{0} 000$ \\
\hline $\begin{array}{l}\text { Chrysoidin } R \\
\text { Rhodamin B }\end{array}$ & 》 & schlecht & $\mathrm{n}: 10000$ & . & $\mathrm{n} / 17500$ \\
\hline Viktoriablan & $n$ & schlecht & $\mathrm{n} / \mathbf{1 0 0 0 0 0}$ & 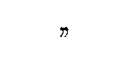 & $\mathrm{n} / 30000$ \\
\hline$+\mathrm{RS}$ & $\pi$ & mittel & n/ 10000 & ? & $\mathrm{n} / 40000$ \\
\hline $\begin{array}{l}\text { Rhodamin } 0 \\
\text { Rhodamin } 3 \mathrm{~B}\end{array}$ & . & nicht & $\mathrm{n}_{/} 10000$ & $"$ & $\mathbf{n} / 10000$ \\
\hline $\begin{array}{r}\text { extra } \\
\text { Diamantfuchsin }\end{array}$ & $n$ & schlecht & n 10000 & ", & n 5000 \\
\hline kl. Krist. & $\therefore$ & nicht & n, 10000 & $\therefore$ & n: 15000 \\
\hline Vilitoriablau $R$ & mittel & gut & n, 5000 & mittel & $\mathrm{n} / 20000$ \\
\hline $\begin{array}{l}\text { Auramin konz. } \\
\text { Toluidinblan }\end{array}$ & 》 & mittel & n: 10000 & gut & $n_{1}: 20000$ \\
\hline Nalachitgrün & 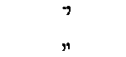 & $\begin{array}{l}\text { gut } \\
\text { mittel }\end{array}$ & $\begin{array}{l}n / 10000 \\
n / 10000\end{array}$ & $"$ & $\begin{array}{l}\text { n } 20000 \\
\text { n } 10000\end{array}$ \\
\hline Vesuvin $4 \mathrm{BG}$ & $\pi$ & mittel & $\mathrm{n} / 10000$ & " & n'30000 \\
\hline $\begin{array}{r}\text { Rhodamin } S \\
\text { extra }\end{array}$ & & fehlt & $\mathrm{n} / 10000$ & & n: 40000 \\
\hline Indazin $\mathrm{M}$ & $"$ & mittel & $\mathrm{n}_{i} \mathbf{1 0 0 0 0}$ & , & n 40000 \\
\hline Acridinrot $3 \mathrm{~B}$ & $n$ & fehlt & n: 10000 & $\nexists$ & Spur \\
\hline $\begin{array}{l}\text { Viktoriablau B } \\
\text { Nilblauchlor- }\end{array}$ & , & mittel & $\mathbf{n} / 5000$ & schlecht & n 3000 \\
\hline hydrat & schwach & gut & $\mathbf{n}: 1000$ & schlecht & $\mathrm{n} / 25000$ \\
\hline Capriblau GON & $n$ & mittel & n 10000 & gut & $\mathrm{n} / 50000$ \\
\hline Nilblausulfat & $n$ & gut & $\mathrm{n} / 10000$ & $\pi$ & $\mathbf{n}_{/} \mathbf{5 0 0 0 0}$ \\
\hline Neutralrot & $n$ & gut & $n: 10000$ & $\eta$ & $\mathrm{n} / 50000$ \\
\hline Bismarckbraun & $\star$ & mittel & $\mathrm{n} / 10000$ & $\forall$ & Spur \\
\hline Methylenblau BB & . & gut & $\mathbf{n}_{1}^{\prime} 10000$ & $=$ & Spur \\
\hline $\begin{array}{l}\text { Methylenblau BX } \\
\text { Methylenblau }\end{array}$ & $n$ & gut & $n / 10000$ & 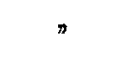 & Spar \\
\hline $\begin{array}{l}\text { rectif. } \\
\text { Methylengrün }\end{array}$ & $n$ & gut & $\mathrm{n} / \mathbf{1 0 0 0 0}$ & $\eta$ & Spur \\
\hline extra gelb 0 & » & gut & n. 10000 & $\pi$ & Spar \\
\hline
\end{tabular}


des milkroskopischen Bildes keinen Aufschluss. Diffuse Färbungen in Zellen sind jedem Beobachter vitaler und supravitaler Färbungsvorgange geläufig. Im allgemeinen werden sie als Zeichen der verlöschenden Vitalităt des Protoplasmas aufgefasst; in der Tat wissen wir ja, dass stark geschädigtes oder abgestorbenes Protoplasma sowohl saure wie basische Farbstoffe stark anzureichern imstande ist.

Ich erinnere hier an die wiederholten Beobachtungen in experimentell geschädigten Organen, in denen bis zu einem gewissen Grade geschädigte Zellen nicht imstande sind, saure Farbstoffe granulär zu speichern, was an einer diffusen Protoplasmafirbung, verbunden mit Kernfärbung, zum Ausdruck liommt.

In meinen Versuchen aber handelt es sich nicht um geschädigte oder gar abgestorbene Zellen, an die die Farbstoffe herantreten; das vollig gesunde Organ wird sorgfiltig dem Tierkörper entnommen, die selbstverstindlich durch die Herausnahme aus dem Organismus verursachte Schidigung ist für alle Versuche annähernà gleich. Wenn also in vielen Fällen eine starke, bald nach dem Versuche einsetzende diffuse Durchtrinkung des Protoplasmas mit dem Farbstoff beobachtet wird, bei anderen Farbstoffen dagegen sich immer wieder nur ganz schwache Diffusfirbung einstellt, so kam aus diesem Ergebnis geschlossen werden, dass der Grad der Diffusfärbung von der Eigenart der verwandten Farbstoffe abhängt.

Unabhángig von dem von mir hier hauptsächlich behandelten Problem der Möglichkeit, Farbstoffe im unfixierten Protoplasma znI Speicherung zu bringen, ist die Frage, inwieweit das Protoplasma unzweifelhaft lebender, d. h. im Verbande des lebenden Tieres befindlicher Zellen diffus gefärbt werden kann. Hierfür sind in der Literatur die Angaben R. Ho ebers (1909), der diffuse Farrbungen mit Rhodaminen beobachtete, vor allem aber von d. Garmus (1912), der nach Eingabe von Rhodamin B bei Fröschen interessante Beobachtungen machte, zu verwerten.

Nach G arm us bleibt eine Granulafärbung bei diesem Farbstoffe stets aus; bei vollkommenem Wohlbefinden des Frosches zeigen dagegen besonders die Zellen der Nickhautdrüsen, die er bei dem lebenden Tiere durch eine sinnreiche Versuchsanordnung beobachten konnte, eine deutliche diffuse Färbung, die nach 24 Stunden wieder verschwindet. Gleichzeitig injiziertes Nethylenblau fürbt in den durch Rhodamin rot gefärbten Zellen die Granula. 
Aus den Beobachtungen von Garmus geht also hervor, dass auch im lebenden Organismus eine reparable Diffusfärbung vorkommt, und ich möchte annehmen, dass bei allen Farbstoffen mit geeigneten physikalisch-chemischen Eigenschaften (siehe darüber unten Seite $521 \mathrm{ff}$.) auch im lebenden Organismus eine diffuse Färbung erreicht werden müsste, wenn nicht manche Faktoren dieser Diffusfärbung hinderlich wären, Falstoren, die in meinen supravitalen Versuchen teils nicht, teils in geringerem Masse in Betracht kommen. Vor allem spielt die Giftigkeit der Farbstoffe eine grosse Rolle.

Aus noch unveröffentlichten Versuchen meiner Schülerin L. K u m mer geht hervor, dass bei einer Reihe nicht granulär sich ablagernder saurer Farbstoffe nur dann ein erbeblicher Grad von Diffusfürbung in den Zellen der Körperparenchyme gefunden wird, wenn die Tiere auch ïnsserlich schwer erkranlit erscheinen. Es muss also, besonders, da mit denselben Farbstoffen im supravitalen Versuche stets starke Diffusfärbung zu crzielen ist (so bei Rose bengale, Erythrosin, Eosin usw.), angenommen werden, dass das lebende Protoplasma bis zu einem gewissen Grade die Fühiglieit besitzt, sich gegen die Farbstoffinvasion zu schlitzen, indem entweder durch Redultion and Oxydation oder durch andere Mechanismen der Farbstoff zerstört wird. Ausserdem lassen diese Versuche den Schluss zu, dass die Farbstoffwirkung mijglicherweise eben durch die diffuse Einlagerung in das Zellenprotoplasma zur vollen Geltung liommt und die in diesem sich abspiclenden wichtigen Lebensworgünge zu stören vermag.

Dass aber Diffusfirbnng und Giftwirkung nicht identisch sind, zeigt schon die oben erwähnte Beobachtung von Garmus; es muss jedenfalls für jeden Farbstoff im vitalen Versuche erst sorgfültig sein Schicksal verfolgt werden, ehe sich ein Urteil gewinnen lässt, wie sich der Farbstoff physikalisch-chemisch zu der speichernden Substanz des Protoplasmas verhält.

Für Protozoen hat E. Nirenstein (1913) eine offenbar sehr eingehende Untersuchung dieser Fragen angestellt, die mir leider erst bei der Niederschrift dieser Arbeit zu Gesicht kam. Er untersuchte an Paramäcien im ganzen 119 Farbstoffe und teilt diejenigen, die eine sichtbare Färbung hervorriefen, ein in Granulafarbstoffe und diffusfiarbende Substanzen.

Unter bestimmten nicht näher charakterisierten Bedingungen sollen sich die kleinen Granula des Paramäcienendoplasmas vergrössern und dann viel besser färbbar werden; sie werden von Granulafarbstoffen auch in Konzentrationen gefärbt, die die kleinen Granula ungefärbt lassen, und ein Teil der diffusfürbenden Farbstoffe färbt nun ausser dem Endoplasma auch die grossen Granula solcher Paramäcien. Es ist aus den kurzen Mit- 
teilungen Nirenste in s nicht ersichtlich, warum die grossen Granula aus den kleinen Granulis hervorgegangen sein sollen; ich kann daher auch nicht übersehen, warum der Autor den Granulis eine endoplasmatische Natur zuschreibt.

Jedenfalls geht aus diesen Angaben hervor, dass auch bei dell Versuchen Nirensteins der Unterschied der beiden Farbstoffgruppen nur graduell, nicht prinzipiell besteht. Diese Bestätigung meiner Befunde ist mir um so wertvoller, weil diese an gänzlich andersartigem Material erhoben wurden. Allerdings bin ich nicht in der Lage, unsere Ergebnisse im einzelnen zu vergleichen, da genaue Angaben in der kurzen Mitteilung Nire nst e in s fehlen.

Ich glaube aber nicht, dass $\mathrm{N}$ ir e $\mathrm{n}$ s t e in mit der Behauptung recht hat, dass alle diffus fürbenden Substanzen nur die Granula nicht stärker färben als das Protoplasma. Ich konnte bei den Versuchen, in denen saure Farbstoffgranula die Stelle von zelleigenen Granulis vertraten, bei allen rein diffus färbenden Stoffen die reine Farbe des sauren Farbstoffes auf dem diffus gefürbten Grunde deutlich erkennen. In diesen Fällen ist eben geradezu weniger Farbstoff in die Granula gezogen, als sich in deren Umgebung ansammelte. Es ergibt sich daraus, dass es anch reine Diffusfürber gibt, die die Granula gänzlich verschmähen.

Aus der Konstanz, mit der bei den einzelnen Farbstoffell in allen Versuchen annahernd der gleiche, in der Tabelle zum Ausdruck gebrachte Grad von Diffusfürbung auftrat, ist zu schliessen, dass wir es jedenfalls mit einer den einzelnen Farbstoffen eigenartigen Funktion zu tun haben. Die auffallende Beziehung, dass die diffusfarbenden Stotfe im allgemeinen die Granula schlecht färben, lässt vermuten, dass eine engere Beziehung zwischen den beiden unterschiedenen Arten der Färbung besteht. Ehe jedoch dieser Schluss gezogen werden kann, muss untersucht werden, ob nicht fur die Diffusfärbung Momente in Betracht zu ziehen sind. die für die Granulafärbung gänzlich ohne Belang sind.

b) Der Grad der Diffusfärbung ist von der Dispersität der Farbstoffe nicht abhängig; das Problem der Zellpermeabilität.

Es könnte ja zunächst die naheliegende Vermutung aufkommen, dass ein Farbstoff um so leichter diffus färben wird, je leichter er in das Zellenprotoplasma einzudringen befähigt ist. Es müssten demnach eben die Faktoren, die die Permeïerfähig- 
keit der Farbstoffe bestimmen, auch für den (Grad der Diffusfürbung ausschlaggebend sein.

Das Problem der Zellpermeabilitat kommt für meine Untersuchungen nur sekundär in Betracht. Bekanntlich hat E. O vert on zum ersten Male nach ausgedehnten Studien den Satz aufgestellt. dass über den Eintritt in das Zellenprotoplasma eine Lipoidhaut entscheide, so dass auch nur solche Farbstoffe in das Zellinnere eintreten, die sich in Lipoiden lösen. Gegen diese Art der Auffassung sind im wesentlichen zwei Arten von Einwänden gemacht worden: es ist nachgewiesen, dass eine grosse Zahl von Farbstoffen in das Zellinnere eindringt, ohne lipoidlöslich zu sein, und andererseits sehr stark lipoidlösliche Farbstoffe in manche Zellen gar nicht, in andere nur schwer permeïeren. (W. Ruhland, R. Hoeber 1914 u. a.) Welche Hilfshypothesen man auch hierbei aufstellen will, die Bedeutung der Lipoide für das Problem der Permeabilitat ist dadurch sehr zweifelhaft geworden. Die andere Gruppe von Einwanden basiert auf der Meinung, dass wohl Lipoide für die Stoffaufnahme und Verarbettung eine grosse Bedeutung besitzen können, dass ihre Bedeutung jedoch nicht in ihrem sitze an einer (Obertlächenschicht zu suchen sei, sondern In ibrer Anwesenheit im Zellenprotoplasma äberhaupt. S. Loewe zeigte, dass $\mathrm{Net}$ hy le $\mathrm{n}$ bl a u aus verdunnten wassrigen Lösungen relativ am meisten, aus konzentrierteren Losungen dagegen relativ weniger ausgezogen wird. Ein kurzer Blick auf meine Tabelle S. 524 zeigt, dass fast alle lipoidlosliche Farbstoffe diese Eigentümlichkeit besitzen. Eine Ausnahme machen hrel die extrem lipoidlöslichen Farbstoffe, wie die Rhodamine und andere. Jedenfalls Laben wir es hier mit einer unter Farbstoffen weitverbreiteten Eigenschaft $z u$ tun, wie auch E. Herzfeld schon zeigte. S. Lo ew e denlit sich demnach den Vorgang der Lipoidiöslichkeit als eine Adsorption des Farbstoffes an die im organischen Lösungsmittel kolloidgelöste Lezithinphase. Er zieht aus dieser Vorstellung den von R. Hoeber nicht akzeptierten Schluss, dass eine Lipoidhaut eher als Speicherungsmedium dienen müsste, jedenfalls also ein Hindernis für ein Eindringen lipoidlöslicher Farbstoffe in das Zellinnere bilden müsste. Ich kann nicht umhin diesen Einwand S. Loewes gegen das Bestehen einer Lipoidhaut als berechtigt anzusehen. Denkt man sich nämlich die Zellipoide an der Oberfläche der Zelle angeordnet, so würden 
sie in der Tat als Speicherungsmedium gerade einem in verdünnter wăssriger Lösung dargebotenen Farbstoffe gegenüber dienen und nur schwer an ein lipoidfreies Zellinnere den Farbstoff abgeben. Denn wenn man zum Beispiel eine intensiv gefärbte Lezithinxylollösung über reines Wasser schichtet, so werden bei den meisten Farbstoffen nur minimale Mengen an das Wasser abgegeben: Der Farbstoff haftet fest am Lipoid. Nur die Gegenwart von besonderen Substanzen in der wissrigen Phase ermöglicht einen stäkeren Austritt des zum Beispiel basischen Farbstoffes ans der Lipoidphase (Beeinflussung durch sauren Farbstoff, s. S. 535). Man müsste also schon im Protoplasmainneren derartige, den Austritt aus der Oberflächenlipoidhaut bewirkende Faktoren annehmen, wenn man sich an das Bestehen einer solchen halten will. Schon von friheren Autoren IW. Ruhland, W. Le peschkin) ist hervorgehoben worden, dass eine Anreicherung lipoidlöslicher Farbstoffe in den Oberflachenschichten der Zellen nicht beobachtet wurde. Lepeschkin aussert sich auf Grund anderer Experimente: "Alles spricht also dafur, dass die chemische Beschaffenheit der oberflächlichen Protoplasmaschicht von derjenigen des inneren Protoplasmas qualitativ nicht verschieden ist. und dass das Protoplasma keine wässrige Lösung darstellt (S. 189)."

Es ist also zum mindesten fraglich, ob mit einer besonderen Obertlichenschicht des Protoplasmas, die für das Permeabilitätsproblem im sinne Overtons Bedeutung bätte, zu rechnen ist. Die Farbstoffexperimente baben in ihrer Gesamtheit keinen Beweis für das Bestehen einer Lipoidhaut erbracht. Damit ist aber die grosse Reihe von Experimenten, die $O$ verton und viele andere Forscher angestellt haben und die die Bedeutung der Lipoidbeziehungen für den Stoffwechsel der Zelle meines Erachtens ausser Zweifel setzen, nicht so hinfällig geworden, wie das wohl von vielen Seiten angenommen wird. Gesetzt den Fall, dass wirklich nicht die Lipoide über den Eintritt der Substanzen in die Zelle entscheiden, so können und müssen sie durch ihre gleichmässige Verteilung in der Zelle eine grosse Rolle für alle Fragen des Stoffaustausches spielen. Da solche Beziehungen zweifellos festgestellt sind, so muss nun morphologisch versucht werden, den Sitz und die Verteilung der Lipoide in der Zelle zu ergründen, um das physiologisch gefundene Tatsachenmaterial für die Lehre von der Zellfunktion zu verwerten. 
Ich sehe an dieser Stelle von den mannigfachen Versuchen, die Verteilung und Anordnung der Lipoidsubstanzen in fixierten Präparaten zu ergründen. ab. Um deren Ergebnisse für die wirkliche Anordnung in lebenden Zellen zu verwerten, ist wie bei allen Fixationsbildern, die Untersuchung der Fixationsmittelwirkung auf die lipoide Substanz erforderlich. Bisher sind einheitliche Resultate nicht erzielt.

Wenn nun die Lipoidhypothese zweifellos dem Verhalten der Farbstoffe nicht gerecht wird, so ist doch $z$ a betonen, dass auch die anderen bisher aufgestellten Theorien nicht völlig befriedigen. Ohne hier genauere Angaben zu machen, möchte ich auf die Schriften W. Rublands $(1912,1914)$ und auf R. Hoebers "Physikalische Chemie der Zelle und der Gewebe" verweisen. in denen das Tatsachenmaterial von verschiedenem Standpunkt aus kritisch beleuchtet ist.

Ob die „Ultrafilterregel“ W. R u h la nds wirklich für alle Zellkategorien gültig ist, ist noch heute nicht zu sagen. Bei PHanzenzellen scheinen ja die Verhaltnisse einfacher zn liegen als im tierischen Organismus. wo wir mit so vielen für ganz spezielle Zwecke angepassten Zellarten zi rechnen haben. Es ist in der Tat schwer moglich, anzunehmen, dass zum Beispiel in dem resorbierenden Dünndarmepithel die so einfachen, für andere Zellarten physikalisch gut deutbaren Speicherungsbedingungen für saure Farbstoffe fehlen, und nur deshalb bei der ganz überwiegenden Mehrzahl derselben keine sichtbare Speicherung von Farbstoff zustande komme. Ich glaube viel eher, dass wir aus den für verschiedene Zellkategorien gefundenen Tatsachen bisher nur den Schluss ziehen können, dass Farbstoffexperimente wenigstens im tierischen Organismus für die Erforschung des Permeabilitätsproblems einen engbegrenzten Anwendungsbereich haben.

Wir müssen uns vorlaufig damit bescheiden, festgestellt $\mathrm{zu}$ haben, dass in den Zellarten, in denen bisher saure Farbstoffe während des Lebens gespeichert aufgefunden wurden, für das Eindringen und für die Speicherung anscbeinend lediglich der Lösungszustand, die Dispersităt, entscheidend ist. Es ist wahrscheinlich, dass diese Zellarten hinsichtlich ibrer Oberflächenbeschaffenheit eine gewisse primitive Stufe bewahrt haben und zwar aus dem Grunde, weil bei Pflanzen diese Abhängigkeit von dem Dispersitätsgrade der zugeführten Substanz viel allgemeiner verbreitet $z u$ sein scheint. Bei vielen anderen Zellarten, in 
denen Speicherung saurer Farbstoffe bisher nicht, oder doch in von dem gewöhnlichen Typus abweichender Weise gefunden wurde, kann es für das Verhalten drei Gründe geben: 1. der Farbstoff dringt ein, wird aber zerstört; 2. der Farbstoff dringt ein, wird aber nicht sichtbar, weil er nicht gespeichert wird; 3 . der Farbstoff dringt nicht ein.

Unter diesen Möglichkeiten ist bisher noch nicht entschieden, und es ist zweifelhaft, ob die Farbstoffe berufen sind, in diesen Fragen eine Entscheidung herbeizuführen.

Veine Untersuchungen wurden nun aber an einer Zellart ausgeführt, der Nierenzelle, für die die Bedeutung der Dispersität wenigstens saurer Farbstoffe für die Permeïerfahigkeit in diese Zellen festgestellt ist, und auch für basische Farbstoffe sind genügend Anhaltspunkte vorhanden (E. Herzfeld 1916), um anzunehmen, dass bei dieser Zellart nur die Dispersität der Farbstoffe über den Grad der Permeïerfähigkeit entscheidet. Es war daher möglich, an dieser Zellart zu entscheiden, ob die Diffusfarbung nur von dem Grade des Eindringungsvermögens, 'das heisst von der Dispersităt del Farbstoftlösung, abhängt.

Cmstehende Tabelle bringt für die verwandten Farbstoffe die Diffusionswerte nach 24,48 und 120 Stunden, wie sie durch Ablesung nach dem $\mathrm{Tr}$ a ubeschen Verfahren in einem $10 \%$ igen Gelatinegel gefunden werden. Es wurde reinste Handelsgelatine in der genannten Konzentration aufgelöst, sorgfültig bis $z u$ neutraler Reaktion mit Sodalösung versetzt, davon je $10 \mathrm{ccm}$ in Reagensröhrchen gebracht und nach dem Erkalten mit $1 \mathrm{ccm}$ einer n/100 Farbstofflösung überschichtet.

Es ergibt sich obne weiteres, dass unter den ausgesprochenen Granulafarbern sowohl wie unter den stark diffus färbenden Stoffen Substanzen von ganz verschiedener Diffusionsgeschwindigkeit enthalten sind Es lässt sich wohl sagen, dass die Intensität der sich ausbildenden Färbung sebr gering ist wenn die Diffusionsgeschwindigkeit gering ist. (Basler Blau $R$ und $B B$, Indazin M), im übrigen aber enthalten sowohl die ausgesprochen diffus färbenden wie die ausgesprochenen Granulafürber Farbstoffe verschiedenster Diffusionsgeschwindigkeit.

Die Werte in der obigen Tabelle stimmen im allgemeinen gut mit den von $\mathrm{Tr} a \mathrm{u}$ be und $\mathrm{K}$ o e hler angegebenen überein; gewisse Abweichungen (Methylgrün, Nalachitgrün) mögen aus der Verschiedenheit des Farbstoff- 


\begin{tabular}{|c|c|c|c|c|c|}
\hline Farbstoff & $\begin{array}{l}\text { Diffusion } \\
\text { nach } \\
24 \text { Std. }\end{array}$ & $\begin{array}{l}\text { fortschrit } \\
\text { nach } \\
48 \mathrm{Std} \text {. }\end{array}$ & $\begin{array}{l}\text { in } \mathrm{mm} \\
\text { nach } \\
5 \mathrm{Tagen}\end{array}$ & $\begin{array}{l}\text { Grad der } \\
\text { Granula- } \\
\text { färbung }\end{array}$ & $\begin{array}{l}\text { Grad der } \\
\text { Diffus- } \\
\text { fārbung }\end{array}$ \\
\hline Malachitgrün & $18(7)^{*}$ & $27(10)$ & 38 (10) & mittel & mittel \\
\hline Safranin $G$ extra & $18(3)^{*}$ & $25(18)$ & $37(29)$ & stark & stark \\
\hline Rhodamin G extra & $17(7)^{*}$ & $22(13)$ & $40(22)$ & fraglich & stark \\
\hline Methylengruin & 17 & 23 & 32 & gut & schwach \\
\hline Methylenblau BB & $17(9)^{*}$ & $25(14)$ & $38(17)$ & gut & schwach \\
\hline Irisamin $G$ extra & 18 & 24 & 38 & fraglich & stark \\
\hline Auramin konz. & 18 & 28 & 42 & mittel & mittel \\
\hline $\begin{array}{l}\text { Capriblau GON } \\
\text { Rhodamin } 3 \mathrm{~B}\end{array}$ & 16 & 23 & 40 & mittel & schwach \\
\hline Rhodamin $B^{\text {extra }}$ & $16(10)^{*}$ & $21(12)$ & $350(19)$ & schlecht & stark \\
\hline (Kahlb.) & $15(8)^{*}$ & $20(8)$ & $34(13)$ & schlecht & sturk \\
\hline Rhodamin s extra & $15(5)^{*}$ & $20(13)$ & $34(20)$ & nicht & miltel \\
\hline Rhodamin 0 & $15(7)^{*}$ & $20(10)$ & $30(13)$ & nicht & stark \\
\hline Bismarckloraun & $1 \overline{0}$ & 23 & 40) & mittel & schwach \\
\hline $\begin{array}{l}\text { Methylgrin } \\
\text { Methylenblau }\end{array}$ & 15 & $2 \tilde{5}$ & 37 & gut & fehlt \\
\hline rectif. & $1+$ & 21 & 41 & gut & schwach \\
\hline Rhorlamin B extra & $1+(5)^{*}$ & $21,11)$ & 30 (19) & schlecht & stark \\
\hline Methylenblau BX & 13 & 17 & 28 & gut & schwach \\
\hline Diamantfuchsin & 13 & 23 & 28 & nicht & stark \\
\hline Akridinrot $3 \mathrm{~B}$ & 13 & 20 & 22 & nicht & mittel \\
\hline Chrysoidin $\mathrm{R}$ & 12 & 25 & 31 & schlecht & stilrk \\
\hline Kristallviolett $6 \mathrm{~B}$ & 11 & 16 & $2 \tilde{0}$ & mittel & stark \\
\hline Rosanilin Base & 11 & 18 & 23 & nicht & stark \\
\hline Methyiviolett 5B & 10 & 16 & 27 & mittel & mittel \\
\hline Neutralrot & $10(4)^{*}$ & $15(6)$ & $22(8)$ & gut & schwach \\
\hline $\begin{array}{l}\text { Vesuvin 4 BG } \\
\text { Nilblauchlor- }\end{array}$ & 10 & 17 & & mittel & mittel \\
\hline bydrat & $8(2)^{*}$ & $15(3)$ & $20(4)$ & gut & schwach \\
\hline Nilblausulfat & 7 & 13 & 17 & gut & schwach \\
\hline Indazin M & 7 & 13 & 19 & mittel & mittel \\
\hline Toluidinblau & 4 & $21(11)^{*}$ & $24(13)$ & gut & mittel \\
\hline Viktoriablau $\mathrm{R}$ & 4 & 6 & 8 & gut & mittel \\
\hline Viktoriablau 4 RS & $1, \tilde{5}$ & $\dot{j}$ & 7 & mittel & stark \\
\hline Viktoriablau B & 2 & 2 & 3 & mittel & mittel \\
\hline Basler Blau R & $(1)^{* *}$ & (2) & (3) & & \\
\hline Basler Blau BB & $(4)^{* *}$ & (5) & (7) & & \\
\hline
\end{tabular}

* Die Klammerzahl bezieht sich auf die Zone dunkelster Färbung.

** Die Klammerzahl bedeutet eine anders gefärbte Vorzone. 
bezuges herrühren. Es ist aber vor allem immer wieder darauf hinzuweisen, dass das Alter der Farbstofflösungen einen grossen Einfluss auf den Dispersitätsgrad besitzt, so dass man eigentlich vor jedem Versuch mit einer längere Zeit nicht benutzten Lösung sich von dem Dispersitätsgrad derselben überzeugen muss.

Kurz zusammengefasst mag man die Bedeutung der Dispersität, des Lösungszustandes der Farbstoffe für die Permeabilität darin erblicken, dass ein gewisses $\mathrm{MaB}$ von Dispersität zweifellos Erfordernis ist, falls ein Farbstoff in das Zellenprotoplasma eindringen soll. Im übrigen lässt sich aus der Beobachtung des Färbungsbildes nicht ohne weiteres ein Rückschluss auf den Grad der Permeïerfähigkeit eines basischen Farbstoffes ziehen. da offenbar noch andere Eintlüsse die Anreicherung dieser Farbstoffe in den Zellen hervorrufen.

Nach den Angaben, die ich oben über die Lipoidtheorie 0 vertons gemacht habe, lag es nahe, der Lipoidlöslichkeit der Farbstoffe Beachtung zu schenken und zu untersuchen, ob nicht diese Eigenschaft vielleicht für die Diffusfärbung verantwortlich gemacht werden könnte. Aus den bisherigen Angaben über die Lipoidlöslichkeit von Farbstoffen, die ich in der Literatur gefunden habe, liess sich ein befriedigendes Bild nicht gewinnen, besonders deshalb, weil im allgemeinen nur bemerkt ist, ob ein Farbstoff lipoidlöslich ist oder nicht; zablenmässige Vergleiche zwischen einer grösseren Reihe von Farbstofien sind bisher nicht gernacht worden. Deshalb und weil die Farbstoffe vielfach trotz Verwendung gleicher Fabrikmarken wechselnde Eigenschaften zeigen, habe ich die Lipoidlöslichkeit der von mir verwandten Farbstoffe untersucht.

\section{c) Die Diffusfärbung ist von den Zellpoiden abhängig.}

Nach anfänglichen Vorversuchen habe ich schliesslich alle Untersuchungen auf Lipoidlöslichkeit mit einer 2\% igen Lösung von Lezithin in Xylol angestellt. Das Lezithinpräparat wurde von $\mathrm{Merck}$ (Darmstadt) bezogen und ist eine wachsartige gelbbraune Masse. In Xylol löst es sich leicht und gibt eine klare gelbe Lösung. Wegen seiner Quellbarkeit ist Lezithin für derartige Versuche in letzter Zeit verpönt; die Löslichkeitswerte, die für die Farbstoffe gefunden wurden, sollen nicht für das Lipoid allein charakteristisch sein, sondern zum Teil auf dem Wassergehalt beruhen. Ich vermag mangels eigener Erfahrungen die Berechtigung dieses Einwandes nicht zu beurteilen. Zweifellos trübt für die physikalisch-chemische Ausdeutung der Versuche die Tatsache das Bild erheblich, dass das käufliche Lezithin keine reine Substanz ist, sondern (nach Nirenstein) durch Zer- 
setzong sowohl Fettsäure als organische Base gelöst enthält. Es ist nach den Ergebnissen dieses Autors sowohl, wie nach denjenigen FauréFremiets (1910) wohl möglich, dass ein gewisser Gehalt an sauren und basischen freien Bestandteilen die Lezithinlöslichkeit für basische und saure Farbstoffe beeinflusst.

Weiterhin halte ich es nicht für wahrscheinlich, dass sämtliche zu den lipoiden Substanzen gerechneten Körper ein dem Lezithin analoges Verhalten zeigen werden.

Die Berechtigung, das Lezithin als Untersuchungsmittel für die von mir bearbeiteten Probleme zu verwenden, ergibt sich einerseits aus der Erwägung, dass wir mit lezithinartigen Substanzen wohl in allen Zellen zu rechnen haben. Ferner werden, so hoffe ich, die Versuchsergebnisse die Wahl dieses Körpers rechtfertigen.

Um ein Urteil über das Verhalten der zur Lntersuchung dienenden Farben zu Lezithinxylol zu bekommen, untersuchte ich zunächst die sogenannte maximale Löslichkeit.

Hierbei kommt folsendes in Betracht:

1. Die gefundenen Zahlen können nicht als absolute Zahlen aufgefasst werden. Sie stellen das Ergebnis von Versuchen dar, in denen in Lezithinxylol die Farbstoffsubstanz bis zur Sättigung in der Kälte aufgelöst wurde. Bei stärkerer Erwärmung liess sich bei allen lezithinlöslichen Farbstoffen eine beträchtlich höher konzentrierte Lüsung herstellen. Die gefundenen Zablen haben ihren Wert nur in der Vergleichungr der fùr die verschiedenen Farbstoffe gefundenen Verte.

2. Bei einer Reihe von Farbstoffen waren die Endkonzentrationen in der Lezithinlösung beträchtlich grösser, wenn der Farbstoff der Lezithinlösung in Wasser gelöst dargeboten wurde gegenuber der direkten Auflösung der Farbstoffsubstanz in Lezithinxylol. Besonders waren es kristallisierte Farbstoffe, bei denen dieses Phänomen beobachtet wurde. Line Verbesserung der Löslichkeit in Lezithinxylol aus Substanz wurde oft schon durch feines Zerreiben der Farbstoffkristalle erreicht.

Die untersuchten Farbstoffe zeigten sebr erhebliche Differenzen in ihrer Löslichkeit in Lezithinxylol; um einen zahlenmüssigen Anhalt für diese Differenzen zu bekommen, wurde grundsätzlich mit aquimolekularen Lösungen gearbeitet. Soweit die Farbstoffe sich gut lösten, wurde eine n/100-Lösung als Ausgangslösung gewählt. Die Konzentration der Lezithinlösung wurde durch Auftropfen auf Fliesspapier und Vergleich dieses getrockneten Fleckes mit einer Skala ermittelt, die durch Auftropfen verschiedener Verdünnungen der wässrigen Lösung gewonnen worden war.

Der Umstand, dass hier die Farbtönung der Lipoidlösung mit der Farbtönung einer wässrigen Lösung verglichen wurde, ist zunächst nicht unbedenklich. Tatsächlich spricht vieles dafür, dass möglicherweise gleich- 
stark gefürbte Lüsungen eines Farbstoffes in Wasser einerseits, in Lezithinxylol andererseits keineswegs die gleiche Farbstoffkonzentration besitzen. Für unsere Fragen aber, bei denen es sich nur um die Möglichkeit des Vergleiches handelt, kann dieser möglicherweise den Versuchen anhaftende Fehler ausser Betracht bleiben, da einerseits die Wertverschiedenheit bei den untersuchten Farben sehr gross ist, andererseits angenommen werden kann, dass der aus dem oben erwähnten Umstand sich ergebende Fehler bei allen Farbstoffen annähernd gleich gross ist.

Die Art der Untersuchung ist andererseits so bequem, dass sie jederzeit leicht durchführbar ist. Aus kolorimetrischen Bestimmungen gewonnene Zahlen können ja ohnehin nur bis zu einem gewissen Grade als zuverlässig gelten.

Hier, wo es sich, wie gesagt, darum bandelt, in erster Linie Vergleichswerte zu bekommen, ist die Methode ausgezeichnet brauchbar.

Ausser diesen direkten Messungen der Löslichkeit der Farbstoffe in der Lezithinxylollösung wurde auch die Fähigkeit der Lezithinlösung geprüft, die Farbstoffe aus wässrigen Lösungen herauszuziehen; es wurde zu diesem Zwecke $1 \mathrm{ccm}$ der Lezithinlosung über $10 \mathrm{ccm}$ einer $\mathrm{n} / 100$ resp. $\mathrm{n} / 10000$ wässrigen Lösung geschichtet. Lässt man die Versuche eine genügend lange Zeit stehen, so erübrigt sich das Umschütteln. In meinen Versuchen liess ich die Lösungen stets 3 Tage aufeinander wirken. Die Ergebnisse der drei Versuchsreihen zur Prüfung der Farbstoffe auf ihr Verhalten zur Lezithinxylollösung zeigt die umstehende Tabelle.

Da in den mir bekannten Arbeiten die Angaben über den Grad der Löslichkeit der Farbstoffe in Lipoiden nur allgemein gehalten sind, lässt sich nur sagen. dass im grossen und ganzen die Ergebnisse mit denen der früheren Untersucher übereinstimmen. Nur zu einigen Farbstoffen müssen einige Bemerkungen angefügt werden.

Valachitgrün ist nach $R$ uhland und Overton sehr wenig löslich in Cholesterin-Öllösung, wo es sich erst bei $70^{\circ}$ ein wenig löst. In Benzol-Lezithinlösung dagegen (Overton) ist es leicht löslich, wie ich auch bestätigen konnte. In starken Benzol-Cholesterinlösungen (40 Teile auf 100 Benzol) löst sich der Farbstoff auch in der Kälte leicht ( $O$ verton).

Nilblausulfat und Nilblauchlorhydrat lösen sich aus $n / 100$ wässriger Lösung recht gut in Lezithinxylol, zu einem grossen Teil aber, wie auch schon andere Beobachter fanden, als freie Base mit rötlicher Farbe. Troptt man von solcher Lezithin- 
xylollösung auf Fliesspapier, so wird der Fleck beim Antrocknen blau, wahrscheinlich durch die Kohlensaure der Luft. Die so schwache Konzentration bei Nilblauchlorhydrat, wenn die Farb-

\section{Löslichkeit basischer Farbstoffe in $2 \%$ igem Lezithinxylol.}

\begin{tabular}{|c|c|c|c|c|}
\hline \multirow[b]{2}{*}{ Farbstoff } & \multicolumn{3}{|c|}{ Konzentration } & \multirow[b]{2}{*}{$\begin{array}{c}\text { Be- } \\
\text { merkungen }\end{array}$} \\
\hline & $\begin{array}{l}\text { bei direkter } \\
\text { Lösung }\end{array}$ & $\begin{array}{l}\text { bei Adsorption } \\
\text { Lösung ven de } \\
\text { n } 100\end{array}$ & $\begin{array}{c}\text { Us wässeriger } \\
\text { Konzentration } \\
\text { n/10000 }\end{array}$ & \\
\hline Rhodamin B (Kahlb) & n 110 & n 100 & $\mathrm{n} / 10000$ & \multirow{31}{*}{$\begin{array}{l}\text { stairlier } \\
\text { als } 1: 100 \\
\text { von der } \\
\text { konz. w. } \\
\text { Lösung }\end{array}$} \\
\hline Rhodamin 0 & $\mathrm{n} / 125$ & $\mathrm{n}: 100$ & $\mathrm{n}_{/} 10000$ & \\
\hline Rhodamin B extra & n 200 & $n: 100$ & $n: 30000$ & \\
\hline Irisamin $G$ extra & n 200 & n 1000 & $n: 15000$ & \\
\hline Safranin G extra & n 200 & n 250 & $n+4000$ & \\
\hline Rhodamin G extra & n 300 & n'100) & $\mathbf{n}: 5000$ & \\
\hline Rhodamin $3 \mathrm{~B}$ extra & n 500 & n 200 & n 5000 & \\
\hline Rosanilin Base & $\mathbf{n} \cdot 600$ & & & \\
\hline Malachitgruin & n 700 & $\mathbf{n} ; 700$ & $\mathrm{n}_{1} \mathbf{1 0 0 0 0}$ & \\
\hline Viktoriablau 4 RS & n. 1000 & n 300 & $\mathrm{n} 40000$ & \\
\hline Chrysoidin $\mathrm{R}$ & ก 2000 & $n 5 k)$ & n 17500 & \\
\hline Capriblau GON & $n 2000$ & $n: 20000$ & $\mathrm{n} / 50000$ & \\
\hline Diamantfuchsin kl. Kr. & n 2000 & n,'5000) & n $1 \overline{10000}$ & \\
\hline Indizin M. & n 2000 & $\mathbf{n} / 5000$ & $n / 40000$ & \\
\hline Auramin konz. & n 2000 & $\mathrm{n} / 1 \overline{1} 00$ & n'20000 & \\
\hline Vesurin $+\mathrm{BG}$ & n 3000 & $\mathrm{n} / 3000$ & $n / 30000$ & \\
\hline Methylviolett 5 B & $n \cdot 3000$ & n 1000 & n 10000 & \\
\hline Kristallviolett $6 \mathrm{~B}$ & $\mathbf{n}_{i}: 3000$ & $n: 2000$ & n 10000 & \\
\hline Viktoriablau $\mathbf{R}$ & $n+5500$ & $\mathrm{n} / 400$ & $\mathrm{n} / 20000$ & \\
\hline Viktoriablau B & $\mathrm{n} / \check{\mathrm{o}} 000$ & & $\mathrm{n} / 3000$ & \\
\hline Nilblausulfat & n. 5000 & $\mathrm{n} / 30000$ & n/50000 & \\
\hline Methylenblau BX & $\mathrm{n} / 5000$ & $n / 15000$ & Spur & \\
\hline Neutralrot & n 10000 & n/25000 & n/50000 & \\
\hline Metbylenblau BB & $\mathrm{n} / 20000$ & $\mathrm{n} / 20000$ & Spur & \\
\hline Rhodamin $S$ extra & $n, 20000$ & $\mathbf{n} 15000$ & $\mathrm{n} / 40000$ & \\
\hline Methylengrün & $\mathbf{n}_{2}: 2000$ & $n, 20000$ & Spur & \\
\hline Tolnidinblau & n. 30000 & n 10000 & $\mathrm{n} / 25000$ & \\
\hline Bismarckbraun & $\mathbf{n} / \overline{0} 0000$ & $\mathbf{n} / 50000$ & Spur & \\
\hline Nilblauchlorhydrat & n. 80000 & n 2000 & $\mathrm{n} / 25000$ & \\
\hline Metbylenblau rect. & Spur & Spur & Spur & \\
\hline Methylgrün 00 & 0 & Spur violett & 0 & \\
\hline Acridinrot $3 \mathrm{~B}$ & 0 & n' 10000 & Spur & \\
\hline
\end{tabular}


stoffsubstanz in Lezithinxylol aufgelöst wird, findet sich nur in Versuchen bei Zimmertemperatur. Schon durch mässiges Erwärmen lassen sich relativ stabile Lösungen bis $\mathrm{zu} n / 4000$ in Lezithinxylol herstellen; die relativ hohe absolute Löslichkeit dieses Farbstoffes in Lezithinxylol zeigt zudem der Versuch aus n/100 wässriger Lösung, wo die Konzentration n/2000 in Lezithinxylol erreicht wird.

Neutralrot war in meinen Versuchen stets mit roter Farbe in Lezithinxylol gelöst; mit Ruhlands (08) Angaben stimmt die relativ schwache Löslichkeit dieses Farbstoffes gegenüber vielen anderen basischen Farbstoffen überein.

Kethylenblau zeigt in den verschiedenen verwandten Marken ein recht verschiedenartiges Verbalten. Metbylenbla u rectif. von Ehrlich ist in Lezithinxylol fast unlöslich; während $\mathrm{M} B \mathrm{BB}$ und $\mathrm{BX}$ etwa mit Nilblausulfat und Neutral rot auf einer Stufe stehen.

Metbylengrün ist nach Overton in Benzol-Cholesterin und Lezithin gut löslich. Ruhland, der die Höchster Marke extra gelblich verwandte, erklärt den Farbstoff für vollkommen lipoidunlöslich. Ich bekam von den Höchster Farbwerken die Marke extra gelblich $O$, die in Lezithinxylol etwa die gleiche Löslichkeit besitzt, wie Methylenblau BB. Bismar ckbra un, das nach $\mathrm{Ruhland}(08)$ praktisch lipoidunlöslich, nacb $\mathrm{Hoeber}$ in Terpentin-Cholesterin eine deutlich gegen reines Terpentin vergrösserte Löslichkeit zeigt, was Rost (11) auch für Lezithinxylol bestätigt, ist in meinen Versuchen zwar sehr schwach, aber deutlich lezithinlöslich. In Xylol ist es nur in geringer Konzentration löslich.

Vethylgrün OO (Grübler) ist mit Methylviolet verunreinigt; dies ist der einzige Bestandteil, der sich im Lipoid löst. Der grüne Farbstoff wird im Lezithinxylol nicht sichtbar. Dies Ergebnis stimmt überein mit der Angabe Rosts (1911), während Ruhland (u8), der offenbar auch mit einem von Grübler bezogenen Präparat arbeitete, angibt, dass der Farbstoff in Cholesterin löslich sei, auch Overtons Angaben lauten so.

Aus diesen kurzen Mitteilungen erhellt, dass die Angaben schwankend sind. Im allgemeinen kann bestătigt werden, dass Cholesterin, wie auch andere Autoren stets betonen, ein schlechteres Lösungsmittel für Farbstoffe ist, als Lezithin. Daraus wird ein 
Teil der unterschiedlichen Angaben erklärlich. Zum Teil handelt es sich aber wohl um die Verwendung verschiedener Präparate.

Das lehren zum Beispiel die hier mitgeteilten Ergebnisse mit verschiedenen Marken von $\mathrm{Rhodamin.} \mathrm{Im} \mathrm{Gegensatz} \mathrm{zu}$ den Jarken B (Kahlbaum), O, 3 B extra, G extra, B extra, die sämtlich $\mathrm{zu}$ den am besten in Lezithinxylol löslichen Farbstoffen gehören, ist Rhodamin S extra auffallend wenig lipoidlöslich. Besonders seine maximale absolute Löslichkeit scheint gering zII sein. während aus verdünnten wässrigen Lösungen eine relativ bedeutende Speicherung in der lipoiden Pbase stattfindet. Rbodamin $S$ extra verhält sich auch, wie gezeigt werden soll, biologisch etwas abweichend.

Trotzdem auf den ersten Blick die Verháltnisse nicht ganz übersichtlich sind, so ist doch unverkennbar. dass eine auffallende Beziehung zwischen der Fähigkeit der Farbstoffe, diffus das Protoplasma $z u$ färben, und ihrer Löslichkeit in Lezithinxylol besteht. Man betrachte hierzu die Tabelle auf s. olı. in deren letzter Reihe die Zahlen verzeichnet sind, die die Konzentration angeben. in der sich die Farbstoffe in $1 \mathrm{ccm}$ $2 \%$ Lezithinxylol bei Hoerschichtung äber eine $\mathrm{n} / 10000$-Farbstofflösung anreichern.

Diese Zahlen wurden gewählt, weil diese Reihe der Reagenzglasversuche am ehesten als Modell für die biologischen Versuche angesehen werden kann, in denen ja anch eine n/10000.Farbstoftlüsung jeweils verwandt wurde. Selbstverständlich sind die im Reagenzglasversuche gewonnenen Werte infolge der schwachen Lezithinkonzentration sehr viel kleiner, als sie ausfallen würden, wenn etwa reines Lezithin zu den Versuchen verwandt worden ware. Bei den verdünnteren Lösungen hat man jedoch den Vorteil. die Abstufung verschieden starker Löslichkeit bei verschiedenen Farbstoffen leichter prüfen zu können. Ich wollte diesen Punkt nur hervorheben, weil die Zellfärbungen in der Tat beträchtlich stärker ausfallen als die im Reagenzglas gefundenen Konzentrationswerte. Fntscheidend für die folgenden Besprechungen ist nur die charakteristische, mit den Reagenzglasversuchen übereinstimmende $A$ bstuf $\mathbf{u} \mathbf{n g}$ der Farbstoffe.

Man beachte: 1. von den stark diffusfärbenden Substanzen, Safranin G extra, Rosanilin Base, Kristallviolett, Irisamin G extra, Chrysoidin R, Viktoriablau 4 RS, Diamantfuchsin kl. Krist., sämtlichen untersuchten Rhodaminen, mit Ausnahme der Marke S extra, sind nur Rhodamin B extra (n/30000) und Viktoriablau \& RS (n/40000) etwas weniger stark löslich in Lezithinxylol. Diese 
beiden Farbstoffe haben aber eine sebr hohe absolute Löslichkeit in Lezithinxylol (n/100 resp. n/300 sind die höchsten gefundenen Werte). Die starken Diffusfärber sind also sämtlich auffallend stark in Lezithin 10 slich.

2. In der Gruppe der mittleren Diffusfärber sind die Werte schwankend. Die Werte für die Lezithinlöslichkeit sind bei der Yehrzahl der Farbstotfe (Viktoriablau R. Toluidinblau, Vesuvin \pm BG. Rhodamin S extra, Indazin V) n/20000-n/40000. Der für diese Gruppe auffallend hohe Wert bei Viktoriablau B erklárt sich daraus, dass die Löslichkeit dieses Farbstoffes in Wasser bei kaltem Ansetzen der Losung sebr begrenzt ist. In dem Reagenzglasversuch wurde die Lösung durch Erwärmen hergestellt. Aus dieser Lösung nimmt das Lezithinxylol reichlich Farbstoff auf. Auch Viktoriablau $\mathrm{R}$ gehört $\mathrm{zu}$ den lipoidlöslichsten Farbstoffen (s. auch Ruhland).

Irh war gleich bei den ersten Versuchen mit diesen Farbstoffen uberl'dscht, dass sie im supravitalen Versuch so rasch und intensiv färben. nachdem $R$ uhl and für Pflanzen ein Eindringen dieser Farbstoffe in tbrede gestellt hatte, und auch $\mathrm{Ho}$ eber zugegeben hatte, dass mit diesen hoch hochkolloidalen Farbstoffen eine relativ schwach ausgedelinte Granulatärbung eintritt.

In meinen Versuchen war regelmässig schon nach 10-15 Hinuten eine ausgedehnte mittelstarke Diffusfärbung, mit gleichzeitig sich ausbildender Granzlafärbung, zu beobachten. Diese Diskrepanz mit vitalen Versuchen liegt zweifeilos an der Hiethodik. Die dem Oruanismus entnommenen Zellen vermógen niclut mehr die dem lebenden Organismus eigenen Schutzkräfte auszuiiben. Würde sich im lebenden Tiere in lebenswichtigen Organen eine so intensive diffuse Protoplasmafärbung ausbilden, so würde in kürzester Zeit eino so enorme Scbädigung Platz greifen, dass das Tier an der Giftwirkung einginge. Nach meiner Exfahrung gibt es nur ganz ausnahmsweise im lebenden Organismus eine Diffusfärbung. Nur, wenn schon äusserlirh das Versuchstier schwer geschädigt ist, ist eine ausgeprägte Diffusfärbung in viru zu erkennen. Im supravitalen Versuche fällt diese Lebensfrage weg. Wir sehen hier nur die physikalisch-chemische Fähigkeit des Protoplasmas. Farbstoffe aufzunehmen oder nicht. Die Funktion der Zelle, iiber die wir in solchen Versuchen Näheres nicht aussagen können, bleibt in diesen Versuchen ausser Betracht.

In diesem Sinne ist für mich das Resultat mit Viktoriabla u $B$ und $R$ un so charakteristischer, als für die schlechte Löslichkeit der Farbstoffe in Wasser das Zustandekommen einer mittelstarken Diffusfärbung erstaunlich ist. Bekanntlich sind die Farbstoffe sehr grobdispers (Ruhland 1912), als solche sind sie zweifellos zum Eindringen in das Protoplasma weniger befähigt als die grosse Mehrzahl der Farbstoffe, die zur Gruppe der starken 
Diffusfärber gehören. Die Lösungen beider Farbstoffe sind aber ansserdem recht instabil, besonders in physiologischer Kochsalzlösung, auf deren Verwendung man natürlich angewiesen ist.

Nach diesen Erwägungen halte ich es für gerechtfertigt, die Viktoriablaufarbstoffe der Gruppe der starken Diffusfärber einzureihen, ihre revera relativ schwächere Jiffusfărbung dagegen auf Rechnung der hohen Kolloïdität und der schlechten Löslichkeit zu setzen.

Die umgekehrte Erwàgung ist bei Acridinnot $3 \mathrm{~B}$ am Platze: dieser Farbstoff ist sehr gut wasserlöslich, leicht diffusibel, aber von begrenzter Lezithinlöslichkeit. Die Bezeichnung „Spur“ soll heissen, dass die Konzentration nicht zu bestimmen war, das Ergebnis des Versuches einer Speicherung in Lezithinxylol aus einer $\mathrm{n} / 100$-Lösung zeigt aber, dass seine Löslichkeit in der Tat nicht gross ist. Die mit diesem Farbstoff gesehene Diffusfärbung ist besonders auffallend, weil eine Granulafärbung in diesen Velsuchen nicht $z u$ erzielen war. Wir werden unten darauf zurückkommen.

3. Sehr klar liegen die Verhältnisse endlich bei der Gruppe del schwachen Diffusfirber. Mit Ausnahme von Nilblauchlorhydrat erreichen diese Farbstoffe keine grössere Fonzentration in Lezithinxylol (bei Überschichtung des Lezithinxylol über n/10000-Losung) als n/5u000. Nilblauchlorhydrat gehört möglicherweise aus denselben Erwägungen wie bei Viktoriablau $B$ und $R$ zu der Gruppe der mittleren Diffusfarber; seine wässrige Lösung ist ausserst instabıl, so dass es schwer ist, einwandtreie, mit den andern Farbstoffen vergleichbare Ergebnisse zu bekommen. Die Farbstoffe also (Capriblau GON, Nilblausulfat, Neutralrot, Bismarckbraun, Methylenblau BB, BX und rectif., Methylengrün extra gelbl. O), sämtlich a usgez eichnete Granulafärber, sind auffallend wenig in Lezithin löslich.

Einige andere in der Tabelle nicht mit aufgeführte Farbstoffe wurden nicht so genau untersucht, ergaben aber keine dem Ergebnis widersprechenden Resultate. Basler Blau $R$ und BB eignen sich zu diesen Versuchen schlecht wegen ihrer schlechten Wasserlöslichkeit, Methylgrün $O O$ ( $G$ r ü bl e r) ist gar nicht lezithinlöslich in meinen Versuchen. Das Ergebnis ist demgemäss besonders interessant.

Vethylgrün $\mathrm{OO}$, aus dessen wässriger Lösung sich nur ein violetter Farbstoff ausschütteln lässt (in schwacher Konzentration, schon von A. Fischer (1899) für eine Beimengung von Methyl- 
violett erklärt), gibt wohl granulare Zellfärbung, auch färbt sich der Kernsaft hellgrün; dass Protoplasma nimmt jedoch nur einen sehr langsam zunehmenden violetten Farbenton an. Dementsprechend ist ja auch Methylviolett ein guter Diffusfärber.

Dies Ergebnis, die völlige Abhängigkeit der supravitalen Diffusfärbung von der Löslichkeit der Farbstoffe in Lezithin, erscheint mir wichtig, besonders deshalb, weil, wie mir scheint, die Bedeutung der Lipoide für die Anreicherung von Substanzen wieder in das rechte Licht gerückt wird. Seitdem mit Sicherheit erwiesen war, dass für die Farbstoffe, mithin wohl auch für ungefarbte Substanzen, die Lipoidlöslichkeit nicht die einzige Bedingung für den Eintritt in die Zelle ist, dass also jedenfalls nicht an der Oberfläche der Zelle eine Lipoidhaut vorhanden sein kann, die ganz allgemein lipoidunlöslichen Substanzen den Eintritt verwehrt, war man vielfach in das andere Extrem verfallen und hatte die Lipoide der Zelle für völlig bedeutungslos für den Stoffaustausch der Zellen angesehen.

Aus meinen Versuchen geht nun zwar nichts hervor, das für eine solche Bedeutung der Lipoide im normalen Zellenleben sprechen würde. Es ist nur, so scheint mir, bestätigt, was von anderer Seite auf Grund ganz andersartiger Überlegungen behauptet worden ist, dass im Zellenprotoplasma in jedenfalls ultramikroskopischer Weise verteilte Lipoide einen wichtigen Anteil an der Zusammensetzung dieses bislang noch unbekanntesten Zellenteiles nebmen.

Ich erinnere hier kurz an die Vorstellungen, die uns E. A lbrecht úber den Bau des Zellenprotoplasmas übermittelt hat. In seinem Vortrag auf dem Anatomentag zu Halle (1902) ausserte er sich zusammenfassend folgendermassen: „1. Es existiert in den Zelleibern aller untersuchten Zellen eine Substanz in diffuser Verteilung, welche a) sehr leicht abspaltbar ist, b) schon durch Aufbewahrung bei Körpertemperatur (Diffusion von Plasma?) $\mathrm{zu}$ Ausbreitungserscheinungen (Myelintropfenbildung) gebracht werden kann, und welche c) bei Unterbindung und Wiederlösung von Nierenarterienligaturen eine diffuse und massenhafte Myelintropfenbildung (Pseudo-Fettdegeneration) in den Nierenzellen erzeugt. 2. Diese Substanz oder Substanzkategorie (Myelin $=$ Lezithin?) erscheint demnach geeignet, die Leichtigkeit $\mathrm{zu}$ erklären, mit welcher sich durch einfache Salzlösungen, ja auch 
durch Wasser das Zytoplasma entmischen lässt. Wahrscheinlich handelt es sich bei der tropfigen Entmischung um eine Bildung minimaler Mengen von Seifen, welche alsdann die entweder nur in Eiweiss gelösten (Quincke) oder mit diesem in äusserst lockerer Verbindung stehenden Myelinsubstanzen zu einer explosionsartigen Ausbreitung an der Oberfläche kleinster dadurch gebildeter Tropfen bringt. Die auffallige Gleichmässigkeit dieser Tropfen weist auf eine ziemlich gleichmässige Verteilung der betreffenden Substanzen in der Zelle hin. (1902.)

In neuester Zeit fübrten kolloidchemische Experimente und C̈berlegungen W. W. Lepeschkins (1913) zu der Auffassung, dass „die Hauptmasse des Protoplasmas eine emulsionskolloide Löstung mit flüssigem Dispersionsmittel darstelle, welche wahrscheinlich zugleich molekular und mehrphasig ist." Das Anftreten von Granulis, Fibrillen und anderen Strukturelementen fasst er als durch eine Dispersionsverringerung bedingt auf. Da L e pescinkin. wie auch schon andere vor ihm, zu einer strikten Ablehnung der Hypothese kommt, es gäbe eine besonders differenzierte Oberflachenschicht des Protoplasmas, so sucht er den friund datür, dass eine Vermischung des P'rotoplasmas mit der umgebenden Flüssigkeit nicht eintritt, in dem Lmstande, dass das Dispersionsmittel des Protoplasmas eine ölartige Substanz sei. Diese Flüssigkeit muss aber Wasser, und zwar molekular. lösen können, weil bei der Plasmolyse und Deplasmolyse Wasser durch das Protoplasma bekanntlich sehr schnell durchdringt; haitte sich aber Wasser dabei im Protoplasma kolloid gelöst, das heisst, wäre es nur in ultramikroskopisch kleine Teilchen zerteilt, so wirden sich die im Zellsaft molekular gelösten Stoffe auch in diesen kolloiden Wasserteilchen gelöst erhalten und mit denselben durch das Protoplasma hindurchgehen, was nicht der Fall ist. (S. 189.)

Lepeschkin stützt sich für diese Auffassung unter anderem auch auf das Verbalten von Farbstoffen. Bei den Farbstoffen, welche gleich schnell durch die Zellenwand diffundieren, hat die Löslich-. keit in ölartigen Substanzen eine grosse Bedeutung: die öllïslichen Farben diffundieren viel schneller durch das Protoplasma. als die in ÖI unlöslichen. (S. 190).

Mit den Lipoiden innig verbunden sind die Eisweisskörper, die das Wasser in Lösung enthalten: "diese Verbindung (der Lipoide mit den Eiweisskörpern) muss flüssig sein und nach. 
ihrer Zersetzung Eiweisskörper in fester Form und in ihnen gelöstes Wasser ausscheiden, so dass die homogene flüssige Grundmasse der lebenden Substanz nach dem Absterben und der stattgefundenen Koagulation als eine porige. schwammartige Masse erscheint, deren Kanäle mit Wasser erfüllt sind und die infolgedessen für alle in Wasser löslicben Stoffe gleich permeabel wird."

An den Ausführungen Le peschkins interessiert für jetzt besonders, dass sie die diffuse Verteilung lipoider Substanzen in der Grundmasse des Protoplasmas sehr wahrscheinlich machen.

Es ergibt sich demnach. dass die Beziehung der Farbstoffe zur Grundmasse des Protoplasmas, die meine Experimente larlegen, sehr wohl so erk]ärt werden kann, dass wir es in diesem Falle tatsächlich mit einem Lösungsvorgange zu tun haben. Die Diffusfärbung wäre demnach nichts weiter als der Ausdruck für die Lösungsfähigkeit eines Farbstoffes in der kolloiden Lösung der Protoplasmagrundmasse

Hierbei ist allerdings die Einschränkung zu machen, dass bisher noch keine befriedigende Erklärung für die Tatsache der Lipoidlöslichkeit gegeben wurde: welcher Art die Lösungsvorgänge in Lipoiden sind, ob es sich dabei um Adsorptionserscheinungen handelt. ob andere chemische oder physikalische Vorginge hierbei mitspielen, ist noch nicht genügend erklirt. So lange wir jedenfalls die Aufnahme der Farbstoffe in die Lipoide als eine Lösıng betrachten, so lange sind wir anch berechtigt, von einer Lösung der Farbstnffe im Protoplasma zu sprechen.

Wie eigene noch unveröffentlichte Versuche und solche meiner Schülerin L. Kum mer zeigen, ist das Parallelgehen von Lezithinlöslichkeit und Diffusfärbungsgrad nicht auf die basischen Farbstoffe beschränkt. In einer Reibe von zirka 40 sauren Farbstoffen, die eine mehr oder weniger starke Löslichkeit in Lezithinxylol besitzen, stufte sich ebenfalls die im supravitalen Versuche gefundene Diffustarbung nach dem Grade der Lezithinlöslichkeit ab.

Ich halte es für noch nicht an der Zeit, in ausgedehnte Erörterungen über das Problem der Farbstoffbindung an Bestandteile des Protoplasmas einzutreten, da bisher wirklich brauchbares ausführlich bearbeitetes Material für diese Fragen noch spärlich vorliegt. Auch hier sind es, so viel ich sehe, nur die Untersucbungen von E. Nirenstein. die zu dem gleichen Problem Stellung nehmen. 
Die Diffusfärbung des Protoplasmas ist nach Nirenstein gebunden an ein Lipoid, das sich wie ein Gemisch von Öl und etwas fettlöslicher Säure (Ölsäure) und Base (Diamylamin) verhält. Das käufliche Lezithin hat eine grosse Übereinstimmung mit dem oben bezeichneten Gemisch, soll diese aber einer durch teilweise Zersetzung bewirkten Verunreinigung durch Säuren und Basen verdanken. Die Übereinstimmung mit meinen Ergebnissen ist evident; mir lieferte die Verwendung von Lezithin die gleiche Beziehung wie Nirenstein das Öl-Ölsäuregemisch.

Die wesentliche Übereinstimmung mit den mir bei Auffindung der Beziehungen zwischen Lipoidlöslichkeit und Diffusfürbungsvermögen der Farbstoffe gänzlich unbekannten Versuchen Nirensteins gibt meinem Schlusse eine erhebliche Sicherheit. Es ergibt sich also, dass in dem Protoplasma in erheblichen Mengen ein Lipoid, vermutlich lezithinartiger Natur, vorhanden sein muss. Dieses Lipoid beeinflusst in supravitalen Versuchen in ausschlaggebender Weise die Farbstoffspeicherung. Aus der Art dieser Farbstolfspeicherung lässt sich schliessen, dass das Lipoid im Protoplasma gleichmässig verteilt ist und nicht etwa seinen Sitz in Teilen der vital färbbaren Granula hat.

Diese Tatsache hat meines Erachtens eine weittragende Bedeutung für die Auffassung des Zellenbaues. Es muss dabei besonders uervorgehoben werden, dass von den zu beobachtenden Farbungserscheinungen ausschliesslich die Diffusfarbung mit dem Grade der Lipoidlöslichkeit parallel geht. Die Tendenz zur Granulafärbung verhält sich geradezu umgekehrt, wie die Angaben der Tabelle auf S. 512 deutlich zeigen. Im wesentlichen lässt sich das so ausdrücken, dass geradezu ein relativ g e ring e r Grad von Lipoidlöslichkeit nötig ist, um eine Granulafärbung in den Zellen zu ermöglichen; vielleicht würde die Granulafärbung bei einem vollständig lipoidunlöslichen Farbstoff, sofern er nur basisch ist, die höchste Elektivität erreichen.

Aus dieser Tatsache schliesse icb, dass Lipoide für das Zustandekommen der Granulafärbung nicht in Betracht kommen, wodurch also auch die Schlüsse ihre Bestätigung finden, die wir aus dem morphologischen Bilde und der Entstehung desselben bei der basischen Granulafärbung zogen. Wie ich weiter unten erörtern werde, befinde ich mich mit dieser Auffassung im 
Gegensatz zu Nirenstein, der sich die Granula ebenfalls aus Lipoiden aufgebaut denkt.

Ich glaube, dass die Ergebnisse meiner supravitalen Versuche, die Beachtung der Diffusfärbung und ibrer Abhăngigkeit von der Lipoidlöslichkeit wohl geeignet sind, zu einer Revision der bisherigen Ergebnisse der Lipoidhypothese von Overton anzuregen. Viele Tatsachen, die früher auf den Lipoidgehalt einer Oberflächenschicht bezogen wurden und so wohl falschlicherweise für das Permeabilitätsproblem verwertet wurden, dürften durch den Nachweis der Beziehungen der lipoidlöslichen Farbstoffe zum intergranulären Protoplasma in ein neues Licht gerückt werden.

\section{Die Lipoidlöslichkeit verschlechtert die Elektivi- tät der Granulafärbung durch basische Farbstoffe.}

Die Angaben des vorigen Kapitels baben abgesehen von der Bedeutung der Zellipoide für die Diffusfärbung auch schon das bemerkenswerte Ergebnis gefördert, dass der Grad der Lipoidlöslichkeit der basischen Farbstoffe gerade im umgekehrten Verhalltnis steht zu der Güte der Granulafärbung, die mit den Farbstottien erzielt werden kann (vergleiche dazu die Tabelle S. 512). Dies Ergebnis ist deshalb wichtig, weil es die erste Feststellung einer allgemeinen Eigenschaft der Farbstoffe ist, die mit dem wechselnden Wert dieser Farbstoffe für eine vitale Granulafürbung parallel geht.

Fast alle guten Granulafarbstoffe sind relativ wenig lipoidlöslich; unter den sehr stark lipoidlöslichen Farbstoffen zeigen nur die Viktoriablaufarbstoffe eine, allerdings mit beträchtlicher Diffusfärbung verknüpfte deutlich ausgeprägte Granulafärbung. Unter den Farbstoffen von mittlerer Lezithinlöslichkeit fallen durch das Fehlen der Befähigung, Granula zu färben, zwei Farbstoffe auf: Rhodamin S extra, Acridinrot 3B.

Die Beziehungen sind so auffaliend, dass man schon durch sie zu dem Schlusse gedrăngt wird, dass die Lezithinlöslichkeit der basischen Farbstoffe dadurch auf die Granulafärbung hindernd einwirkt, dass die Farbstoffe sich zum grössten Teile in dem intergranulären, lipoidhaltigen Protoplasma einlagern. Dieser naheliegende Schluss liesse aber den Einwand zu, dass Färbung des Protoplasmas und Granulafärbung keineswegs so innig mit- 
einander verknüpft seien, sondel'n dass beide Erscheinungen nur nebeneinanderhergehen. Ausserdem mussten die auffallenden, oben erwähnten Ausnahmen noch erklart werden.

Ich suchte daher die nach den bisherigen Feststellunger vermuteten Zusammenhänge zwischen den Lipoiden des I'rotoplasmas und den Granulasubstanzen im Reagenzglas nachzuahmen und ging dabei von folgender Überlegung aus: Ler Farbstoff. der in dem Aussenmedium enthalten ist, muss jedenfalls. ehe er an die Zellgranula kommt, die Grundmasse des Protoplismas durchdringen. Hierbei kommt er in innige Berührung mit den gleichmässig in der Zelle verteilten Lipoiden. Ist er in diesen sehr gut löslich. so wird er, bevor er die Zellgranula erreichen kann, im Protoplasma haften bleiben: er wird diffus färben. Auf die Lokalisation des basischen Farbstoffes wirkt aber andererseits ohne Zweifel der Zug der sauren Substanzen em. die in den Zellgranulis ihren Sitz haben. Ist dieser Zug stark. haftet andererseits der Farbstoff nicht $\mathrm{zu}$ stark an den Zellipoiden. so wird es zu einer vorwiegend granulïren Färbung kommen.

Besteht wirklich dieser Widerstreit zwischen dem Eintuss der Lipoide und den sauren Granulasubstanzen, so kommit es darauf an, zu untersuchen, wie der in Lezithinxylol geloste basische Farbstoff sich za einer mit Lezithin nicht mischbaren Süure verbält. An Stelle der unbekannten Säuren in den normalen Zellgranulis konnte ich in die wässrige Phase gewisse Mengen saurer Farbstoffe geben, da ja die Übereinstimmung salurer Farbstoffgranula mit den normalen Zellgranulis bei Färbung durch basische Farbstoffe erwiesen ist.

Demnach schichtete ich Lezithinlösungen basischer Farbstoffe von bekannter Konzentration über bestimmte Quantitüten in Wasser gelöster saurer Farbstoffe; bemerkenswerterweise wird ein Teil der basischen Farbstoffe durch die Anwesenheit des sauren Farbstoffes in der wässerigen Phase aus dem Lipoid ausgezogen, wâhrend andere viel weniger oder gar nicht beeinflusst werden. werden:

Für die Art meiner Versuche mag folgendes Beispiel angeführt

Auramin, das mit Wasserblau im Verhältnis 2:1 ausgeflockt wird. wurde durch Erwärmen in Lezithinxylol bis zur Konzentration $n_{/} / 500$ gelöst; von dieser Lösung wurden je $1 \mathrm{ccm}$ über a) $1 \mathrm{ccm} \mathrm{Aq.} \mathrm{dest.,} \mathrm{b)} 1 \mathrm{ccm}$ n/1000 Wasserblaulösung geschichtet. Nach gleichmässigem Umschütteln 
beider Gläschen war die Endkonzentration der Lipoidphase bei a) auf $n, 600$. bei b) dagegen auf $n_{i} 1100$ gesunken. Die wässerige Phase im Gläschen a hatte einen hellgelben Ton angenommen; im Glaschen $b$ dagegen war die Wasserblaulösung vollständig ausgefällt Auramin war also durch das Vorhandensein des Wasserblau in der wässrigen Phase stark aus der lipoiden Plase herausgezogen worden.

Rhodamin S extra, das mit, Trypanblau, dem gegenüber sich Auramin ebenso verhält wie gegeniiber Wasserblau, sich auch im Verhältnis 2:1 gut ansflockt, wird aus einer n:2000-Lösung in Lezithinxylol durch einen Gehalt von n'2000 Trypanblau in der wiisserigen Phase aus dem Lezithin nicht stärker ausgezogen als durch die reine wässrige Phase. In beiden Fällen stellt sich die Endkonzentration n.3000 ein.

In dieser Weise fand ich, dass alle guten Granulafirber aus der Lezithinlösung dureh Trypanblau a usgezogen werden, dass dagegen die ausgesprochenen $D$ iff usfärber durch die tnwesenheit von Trypanblau nicht beeinflusst werden.

Von Farbstoffen, die mit Trypanblau bei Vermischung beider Farbstoffe in wässriger Lósung im Verbältnis 1:1 reagieren, werden durch Trypanblau aus dem Lipoid herausgezogen: am stärksten Neutralrot, gut, aber weniger reichlich Toluidinblau und Malachitgrün, gar nicht Diamantfuchsin.

Dies Verhalten entspricht vollständig der Tatsache, dass Neutralrot der beste Granulatürber, 'Toluidenblau und Malachitgrün neben mittelstarker Diffusfärbung gute Granulafïrbung, Diamantfuchsin bei starker Diffusfärbung sehr schlechte (xiranulafürbung ergibt.

2:1 fällen sich mit Trypanblan Auramin, Rhodamin Sextra, Safranin Gextra, Methylviolett; Auramin und Methylviolett werden etwa so stark durch Trypanblan dem Lipoid entzogen, wie Malachitgrün, Safranin in kaum eben erliennbarer Menge. Rhodamin \$ extra gar nicht.

3:1 fällen sich mit Trypanblau Kristallviolett, Methylengrün, Rhodamin B extra. Kristallviolett und Methylengrün werden wieder mittelstark durch Trypanblau der Lezithinlösung entzogen, während Rhodamin B extra durch dieselbe vollkommen unbeeinflusst bleibt.

Von der Mitteilung weiterer Versuche, die die hier gemachten Angaben bestätigen. sehe ich ab. Auch die zahlenmässig von mir bestimmten Werte glaube ich nicht mitteilen zu müssen, weil sie nur für den Untersucher Wert haben. Ich will hier nur zur Orientierung angeben, dass die Konzentrationsabnahme der lipoiden Phase in dem günstigsten Fall (Neutralrot) von n 3000 nach n/10000 lag. Werte, die beim Auftropfen auf Fliesspapier und Vergleich mit einer Fleckenskala sehr leicht annähernd genau bestimmt werden können. Bei den mittelstark abnehmenden Farbstoffen (Malachitgruin, Lethylviolett usw.) betrug die Konzentrationsdifferenz etwa n/2000 bis n 4000 , also einen Abfall auf die ${ }^{1}$ a Konzentration, was auch noch sehr scharf bestimmt werden kann. 
Die mitgeteilten Versuche haben mich überzeugt, dass die in dem Modellversuch untersuchte Beziehung zwischen einer lipoiden Phase und einer wässrigen sauren Phase in der Tat die Verbältnisse wiedergibt, die in der Zelle zwischen Grundmasse des Protoplasmas und den durch basische Farbstoffe farbbaren Zellgranulis hergestellt sind.

Damit ist der Weg angebahnt, um $z u$ einem befriedigenden Verständnis der Tatsache zu kommen, warum unter einer grossen Reihe basischer Farbstoffe die einen Granula färben, andere diffius färben, noch andere endlich sowohl Granula wie diffus färben. Ehe ich aber dazu übergehe, zusammenfassend darzulegen, wie der ganze Vorgang der vitalen Farbung nach diesen Untersuchungen aufzufassen ist, muss ich noch auf einige Punkte hinweisen, die möglicherweise der Beweiskraft meiner Versuche entgegengehalten werden könnten.

Ich habe zunächst nur gezeigt, dass bei basischen liarbstoffen von gleicher Fällungskraft ein stärkeres Haften an der lipoiden Phase die Reaktion mit dem sauren Farbstoffe behindert, dass es also darauf ankommt, ob die Zugkraft des Lipoids oder die Zugkraft der Saure grösser ist, um eine Granulafärbung zu befördern oder zu verhindern.

Es könnte ja aber auch sein, dass an und für sich die stärkere Lipoidlöslichkeit ein Grund wäre für das Versagen der Granulafarbung. Demgegenüber ist aber auf die Farbstoffe hinzuweisen, die trotz relativ geringer Lipoidlöslichkeit schlecht oder gar nicht $\mathrm{zu}$ einer Granulafürbung zu bringen sind. Hierhin gehört Rhodamin $\mathrm{S}$ extra. Ebenso ist das Ergebnis bei Acridin rot $3 \mathrm{~B}$ zu erklären: dieser Farbstoff reagiert ganz auffallend wenig mit sauren Farbstofien (mit Trypanblan z. B. 1:9). Es ist klar, dass nach dem oben Gesagten eine so geringe Fällungskraft selbst bei geringerer Lipoidlöslichkeit keine erkennbare Anreicherung des Farbstoffes in den Zellgranulis bewirken wird.

Es zeigt sich aber auch weiter, dass unter Farbstoffen, die sich annähernd gleich gut in Lezithinxylol lösen, gute Granulafärber und schlechte Granulafärber vorkommen. In diesen Fällen werden die guten Granulafärber durch Trypanblau aus der lipoiden Phase herausgezogen, die schlechten dagegen kaum erkennbar oder gar nicht: Auramin, Methylviolett, Kristallviolett, Malachitgrün werden 
gut aus der lipoiden Phase herausgezogen, sind demgemäss trotz hoher Lipoidlöslichleit gute Granulafärber, das heisst, die Granula. besonders wenn sie sauren Farbstoff enthalten, werden zuerst gefärbt, während Diamantfuchsin weder aus der lipoiden Phase extrahiert wird, noch Granula färbt.

Das Ergebnis der supravitalen Färbung mit basischen Farbstoffen wird also bestimmt durch das Konkurrieren der diffus im Zellenprotoplasma verteilten Lipoide mit den granulär abgelagerten sauren Substanzen (vermutlich Eiweisse) um den Farbstotf.

An dieser Deutung, die sich mir aus den Versuchen mit allen daraufhin geprüften basischen Farbstoffen ergeben hat, halte ich auch fest angesichts der mir erst nachträglich bekannt gewordenen Mitteilungen E. Nir ensteins (1913). Dieser Autor, der bezüglich der Diffusfärbung und ihrer Abhăngigkeit von der Lipoidlöslichkeit zn ganz ähnlichen Ergebnissen kam wie ich, dehnt seine Theorie der vitalen Färbung auch auf die Färbung der Granula aus. Er nimmt an, dass die Färbung der Granula ebenfalls auf einem Lösungsvorgange der Farbstoffe beruht. Diese Annahme ist aber; wenigstens in dem Bericht über den Vortrag, der mir vorliegt, in keiner Weise gestützt. Im Gegenteil hatte schon Nirenstein (1905) ebenso wie schon viele andere Forscher vor ihm gezeigt, dass die Farbung der Nahrungsvakuolen bei Protozoen von dem Säuregehalt abhängig ist, dass der Inhalt der Nahrungsvakuolen nur so lange mit basischen Farbstoffen färbbar ist, so lange in der Vakuole saure Reaktion herrscht, ein Ergebnis also, das vielmehr für die Deutung der Färbung als einer Reaktion des Farbstoffes mit dem Vakuoleninhalt spricht, als für die Annahme eines Lösungsvorganges Ich möchte zudem noch einmal darauf hinweisen, dass die genaue morpbologische Betrachtung die Frage zu Gunsten einer kolloidchemischen Reaktion entschieden hat.

$\mathrm{N}$ irenstein fand nun, dass Farbstoffe nur dann imstande sind, die Granula stärker zu färben als das übrige Protoplasma, wenn ihre Löslichkeit in Öl durch den Zusatz einer fettlöslichen Säure (Ölsäure) verstärkt wird. Dies ist der Fall nur bei basischen Farbstoffen, während saure und indifferente Farbstoffe nur durch den Zusatz einer fettlöslichen Base (Diamylamin) zu Öl in ihrer Löslichkeit beeinflusst werden. Ich halte es durchaus für richtig, dass die von $\mathrm{Ni}$ ren stein gefundene Parallelităt besteht, sehe aber nicht, wie durch diese Auffassung die Besonder- 
heit der Granulafürbung gegenüber der diffusen I'rotoplasmafärbung erklärt wird.

Die Beeintlussung der Granulafärbung durch den Säuregehalt der Granula halte ich mit Nirenstein für erwiesen. Nirenstein musste aber zu Fehlschlüssen kommen dadurch, dass er die Eigenschaften der Lipoidlöslichkeit und der Säurebeeintlussung nicht getrennt prüfte; er verwischte das Bild geradezu dadurch, dass er eine fettlösliche Säure zu seinen Experimenten wilhlte. Er musste darum zu der Vorstellung gelangen, dass auch in den Granulis stark saure Lipoide die stärkere Färbung bedingen. Nachdem aber erwiesen ist. dass auch an sicher nicht lipoiden (sauren Farbstoff-) Granulis die basische Vitalfarbung gelingt, ist die ganze Vorstellung Xirensteins bezüglich der Granula hinfällig.

In der Tat glaube ich, dass bisher einzig das oben S. 5:34 beschriebene Modell den tatsächlichen Verhältnissen nahe kommt. Der Farbstoff, der in die Zelle eingetreten ist, sieht sich einem Milieu gegenuber, das zu einem grossen Teil aus Lipoiden aufgebaut ist. Diese Lipoide sind im Zelleib diffus, möglicherweise als Dispersionsmittel für die emulsionsartige Masse des Protoplasmas (Lepeschkin 1913), verteilt. In ihm lösen sich die Farbstoffe nach Massgabe ihrer Lipoidlöslichkeit (nach Nirenstein gemessen in einem Öl, dem etwas Ölsiure und etwas Diamylamin zugesetzt ist, nach mir in Lezithinxylol). Ob ein Farbstoff fähig ist, sich in stärkerem Masse an die im Zelleib suspendierten sauren Granula anzulagern băngt $a b: 1$, von der Fällungskraft des basischen Farbstoffes (basische Farbstoffe mit minimaler Affinităt wie Acridinrot $3 \mathrm{~B}$ färben schlecht) oder 2. bei gleicher Fällungskraft zweier basischer Farbstoffe davon, ob die Fällungskraft die Lipoidlöslichkeit überwiegt oder nicht.

Die Granulafärbung einerseits, die diffuse Protoplasmafärbung andererseits sind also nicht etwa graduell voneinander verschieden, wie E. Nirenstein meint, sondern sind zwei prinzipiell voneinander streng $\mathrm{zu}$ sondernde Vorgänge, die sich gegenseitig beeinflussen. Die diffuse Färbung ist das Ergebnis einer physikalischen Lösung der Farbstoffe in den Lipoiden des Zelleibs, die Granulafärbung kommt durch die Reaktion entgegengesetzt geladener Kolloide zustande und wird durch einen stärkeren Grad von Lipoidlöslichkeit behindert. 


\section{Zusammenfassung und Zusätze.}

\section{a) Morphologische Bewertung der Granula auf Grund vor-} stehender Versuche.

1. Die Granula, die mit basischen Farbstoffen vital und supravital furbbar sind, sind in der Regel präformierte, aber nicht integrierende Bestandteile des Zelleibs.

Es ist wohl möglich, aber nicht sicher erwiesen, dass im Protoplasma gelïst gehaltene Eiweissstoffe durch die Einwirkung basischen Farbstoffes ausgefällt werden, wodurch neue Granula entstehen könnten. Prinzipiell unterschiede sich diese Möglichkeit nicht von der Fällung saurer Inhaltsmassen an der Oberfläche der Granula oder im Inneren derselben, ein Voryang. der von mir genau beobachtet worde.

Trotz der vielfach (A. Fischel 1901, E. Gol dmann 1909, 1912) betonten Tatsache. dass die Granula Iange Zeit. unverändert im Zelleib erhalten bleiben, sind die Granula keine unverinderlichen, zur Zelleibsstruktur zugehörigen Bildungen. Thre lange Lebensdauer hängt mit der Art ilures Zustandekommens zusammen. Als anodische Substanzen werden sie durch Dispersitätsverminderung solange in den Zellen deponiert, bis die zuströmende Flüssigkeit keine neue Zufuhr solcher Substanzen mehr bringt. Das durfte im normalen Zelleben niemals eintreten. Deswegen sind stets derartige Granula aufzufinden. Dass sie aber nicht konstant sind, lebrt die Granulabildung. die experimentell durch die Zufuhr saurer Farbstoffe hervorgerufen werden kann. Solche Granula entstehen und werden um so stiirker konzentriert, je linger eine gewisse Konzentration des Farbstoffes der speichernden Zelle zuströmt. Hört die weitere Zufuhr auf, so wird durch Dispersitätserhöhung der grö̈sste Teil der Granula zum Abblassen und zu allmählichem Schwunde gcbracht. Hier haben wir also den Beweis für die Vergïnglichkeit nnd Veründerlichkeit des Granulainhaltes.

2. Es ist bisher noch nicht aufgeklärt, unter welchen Bedingungen sich Plast os omen, das heisst integrierende $\mathrm{Be}$ standteile des Zelleibes. färben.

Bisher fehlt eine systematische Erforschung dieser Fragen so gut wie vollständigg. Die zur Plastosomenfärbung angewandten Farbstoffe sind stark lipoidlöslich (Janusgrün. Mlethylviolett, Dahlia). Die Färbung geht, soviel aus der Literatur ersichtlich, langsam vor sich im Gegensatz zu der schnellen Granulafärbung. Dass tiefgreifende postmortale Veränderungen notwendig sind, um Plastosomen zu färben, ist noch nicht auszuschliessen.

\section{b) Physiologische Bewertung der Granula.}

1. Die Granula haben an der Farbstoffaufnahme keinen aktiven Anteil.

Die Granulafärbung ist zurückzuführen auf den Gehalt der Granula an sauren Substanzen. Darüber hinaus haben die Granula keinen Anteil an der Färbung. 
Dieser Auffassung bereitet auch die Möglichkeit der Oxydasefärbung an diesen Zellgranulis keine Schwierigkeit. Die Oxydasereaktion verläuft vollständig ebenso wie die supravitale Granulafärbung, wie auch schon von anderer Seite hervorgehoben wurde. Der Oxydasefarbstoff wird sicherlich im intergranulären Protoplasma gebildet; den Granulis gegenüber zeigt er das Verlıalten eines gewöhnlichen basischen Farbstoffes. Auch an sauren Farbstoffgranulis gelingt die Oxydasereaktion! Die Oxydase hat also in den Zellgranulis ihren Sitz nicht.

2. Die Granula sind einer ständigen Veränderung unterworfene kuglige Einschlüsse des Protoplasmas mit einem flüssigen Inhalt, der vermutlich eine wässrige Lösung kolloid gelöster Stoffe (Eiweisse, Kohlehydrate, vielleicht auch Fettsäuren) darstellt.

In die Granula können sich Pigmente einlagern. ebenso wie kolloide saure Farbstoffe. Die Zahl der Granula kann vermehrt werden je nach der Zufuhr -granulafüliger" Substanzen.

Dass der Inhalt der Granula in der Regel Hlüssig ist, zeigt die Beobachtung Brownscher Molekularbewegung, feiner Körnchen in, ausgeflockten ${ }^{\star}$ Granulis. Nur bei extremer Konzentration, vollständiger Ausflockung, kann der Inhalt des Granulums fest werden.

Über die Natur der Inhaltsstoffe ist bisher nichts Bindendes auszusagen. Er ist jedenfalls sehr veränderlich. Dass Fettsüuren (Nirenstein) sich an dem Aufbau der Granula beteiligen, ist nicht völlig auszuschliessen; jedenfalls ist die Anwesenheit von lipoiden Substanzen für die Fürbbarkeit unwesentlich. die Anwesenheit von Süuren notwendig.

Das Bestehen der Granula in der vermutlich wesentlich aus Lipoiden zusammengesetzten Lösung des Protoplasmas spricht für den Aufbau der Granula aus einer mit Lipoiden nicht mischbaren Flussigkeit. Wahrscheinlich sind die Granula also Tröpfchen einer wässerigen Lösung.

3. Die Granula können wahrscheinlich entgiftend wirken durch Bindung basischer Gifte. Möglicherweise kommt dieses Verhalten bei der Einwirkung der Alkaloidbasen in Betracht.

Alle wesentlichen Lebensprozesse spielen sich wahrscheinlich in der Grundmasse des Protoplasmas ab; hier hat zum Beispiel die Oxydase ihren Sitz usw. Die Granula verhalten sich bei der vitalen Färbung passiv. Die Giftwirkung eines Farbstoffes entfaltet sich vermutlich um so stïrker, je mehr der Farbstoff entweder infolge starker Lipoidbeziehungen oder nach Absättigung der Granula sich in die Grundmasse des Protoplasmas einlagert. Vermehrt man die Menge der sauren Zellorte im Körper durch granuläre Ablagerung saurer Farbstoffe, so wird die Giftwirkung basischer Farbstoffe abgeschwächt (s. E. Herzfeld 1916).

\section{c) Die Färbung der Granula.}

1. Die Färbung der Granula ist bewirkt durch eine Reaktion des basischen Farbstoffes mit der in den Granulis vorhandenen kolloidalen Säure. 
Die Bedeutung von sauren Kolloiden und Lipoiden.

Ob die Reaktion unter Salzbildung verläuft oder eine kolloidchemische Fällung darstellt, ist nicht entschieden. Die Vorgänge bestätigen jedenfalls die $\mathrm{Heidenh}$ a in sche Ansicht von der Umsetzung zwischen Eiweisskörpern und Anilinfarben. Nur muss auch in diesen Füllen die physikalisch-chemische Forschung noch entscheiden, ob es sich um Kolloidfällung oder typische Salzbildung handelt.

Ein Lösungsvorgang kommt nicht in Betracht; jedenfalls ist er stets sekundär beteiligt und analog der Lösung des Fällungsproduktes im Überschuss einer der beiden reagierenden Komponenten (saurer und basischer kolloidaler Farbstoff). Eine spezifische Löslichkeit der Farbstoffe in einer lipoiden Substanz (A. Pappenheim, R. Hoeber, E. Nirenstein u. ঝ.) kommt für die Granula nicht in Betracht.

2. Die Färbung der Granula wird beinflusst durch die im intergranulären Protoplasma (Grundmasse) vorhandenen Lipoide.

Entscheidend ist das Verhiltnis zwischen Lipoidbeziehung und Fällungskraft jedes basischen Farbstoffes. Ist bei zwei gegebenen basischen Farbstoffen die Lipoidlöslichkeit gleich gross, wird derjenige die Granula besser färben, dessen Fällungskraft die Lipoidlöslichkeit überwindet. Wenig lipoidlösliche Farbstoffe mit starker Fällungskraft sind gute Granulafärber, stark lipoidlösliche Farbstoffe mit schlechter Fällungskraft sind vorwiegend Diffusfärber.

\section{Literaturverzeichnis.}

1. Albrecht, E., 1902: Artefacte zur Cytologie. Verh. Anat. Ges., Halle a. S., 211-213.

2. F is chel, A., 1901: Untersuchungen über vitale Fürbung. Anat. Hefte. Bd. 16, $417-519$.

3. Derselbe, 1910: Vitale Färbung. Enzylil. d. mikr. Techn. 2. Aufl., כ89-601.

4. F is cher, A., 1899 : Fixierung, Färbung u. Ban d. Protoplasmas. Jena, 1899.

5. Garmus. A., 1912: Fortgesetzte Untersuchungen über die physiologische Permeabilität der Zellen IV. Zeitschr. f. Biol. N. F. 58, 185-236.

f. G oldmann, E., 1909: Die äussere and innere Selretion des gesunden Organismus im Lichte der ,vitalen "Färbung. Tübingen, Laupp.

7. Derselbe, 1912: Neue Untersuchungen über die äussere und innere Sekretion des gesunden und kranken Organismus im Lichte der "vitalen " Färbung. Tübingen, Laupp.

8. Derselbe, 1913: Vitalfärbung und Zentralnervensystem. Abh. d. Kgl. Preuss. Akad. Wissensch. 1913, Physik.-math. Cl.

9. Heidenhain, M., 1911: Plasma und Zelle, II. v. Bardelebens Handb. d. Anat. 8, 434-487 (dort weitere Literatur).

10. Herzfeld, E., 1916: Über die Natur der am lebenden Tier erhaltenen granulären Färbungen bei Verwendung basischer und saurer Farbstoffe. Anat. Hefte, Bd. 54, 447-523.

11. H o e ber, R., 1901 : Über die Resorption im Darm. Pflüg. Arch. 86. 199-214.

12. Derselbe, 1909: Die Durchlïssigkeit der Zellen für Farbstoffe. Biochem. Zeitschr. 20, 56-99.

Archiv t. mikr. Anat. Bd. 90. Abt. I. 
542 Wilhelm von Moellend orf $f$ : Die Bedeutung von sauren Kolloiden.

13. Derseibe, 1914: Physikalische Chemie der Zelle und der Gewebe, 4. Aufl. (dort weitere Literatur).

14. Derselbe und Nast, 0., 1913: Weitere Beiträge zur Theorie der Vitalfärbung. Biochem. Zeitschr. 50, 418-437.

15. H of er, Br., 1890: Experimentelle Untersuchungen über den Einfluss des Kernes auf das Protoplasma. Jen. Zeitschr. f. Nat. 24, 105-175.

16. Laguesse, E., 1912: Méthode de coloration vitale des chondriosomes par le vert Janus. Compt. rend. Soc. Biol. Paris, 73. 150-153.

17. Lepeschkin, W. W., 1913: Über die kolloidchemische Beschaffenheit der lebenden Substanz und über einige Kolloidzustände. die für dieselbe eigentümlich sind. Kolloidzeitschrift 13, 181-192.

18. Loewe, S., 1912: Zur physikalischen Chemie der Lipoide, I--IV. Biochem. Zeitschr. $42,150-218$.

19. Michaelis, L.: Die vitale Fürbung, eine Darstellungsmethode der Zellgranula. Arch. mikr. Anat. 55, 558-575.

20. M o ell end orf f, W. vo n, 1918: Zur Morphologie der vitalen Granulafärbung. Arch. mikr. Anat. (dort weitere Literatur.

21. Nirenste in, E., 1905: Beiträge zur Ernährungsphysiologie der Protisten. Zeitschr. allg. Physiol. 5, 435-5̃10.

22. Derselbe, 1913: Das Wesen der Vitalfärbung. Verb. Ges. Deutsch. Naturf. a. Aerzte, 85. Vers. Wien 1913, II. 2, \$. 8-78.

23. Orerton, E., 1900: Studien über die Autuahme von Anilinfarhen durch die lebende Zelle. Jahrb. wiss. Bot. 34, 669-701.

24. Pappenheim. A., 1911: Über die Vitalfürbung und die Natur der vital fürbbaren Substanzen der Blutkörperchen. Fol. haemat. 12, Arch. 289-301 (dort weitere Literatur des Autors).

25. Pelet-Jolivet, L., 1910: Die Theorie des Fïrbeprozesses. Dresden, Steinkopff, (dort ausführliche Erörterung der physikalisch-chemisehen Eigenschaften der Farbstoffe mit Literaturangaben).

26. Plato, J., 1900: Üher die vitale Färbbarkeit der Phagozyten. Arch. mikr. Anat. 56, 868-917.

27. Rost, Fr, 1911: Über Kernfärbung an unfixierten Zellen und innerlaalb des lebenden Tieres. Pfüg. Arch. 137, 359-421.

28. Ruhland. W., 1913: Zur Kritik der Lipoid- und der Eltrafltertheorie der Plasmahaut nebst Beobachtungen über die Bedeutung der elektrischen Ladung der Kolloide für ihre Vitalaufnahme. Biochem. Zeitschr. jt, S. 29 (dort weltere Literatur des Autors).

29. Seyewetz, A., 1900: Sur les combinaisons des matières colorantes acides avec les matières colorantes basiques. These de Lyon.

30. Teague, O. und Buxton, B. H., 1907: Die Agglutination in physikalischer Hinsicht, IV. und V. Zeitschr. physik. Chem. 60, 469-506.

31. Traube, J. und Koehler, F., 1915: Über Farbstoffe. Intern. Zeitschr. physik.-chem. Biol. 2, 197-226.

32. Vaubel und Bartlet, 1906: Über die Verwendung von Metbylenblau zur quantitativen Bestimmung von Sulfosüuren aromatischer Amidound Oxyverbindungen. Zeitschr. f. Farbenind. 5, 102. 